\title{
Albedo Properties of Main Belt Asteroids Based on the Infrared All-Sky Survey of the Astronomical Satellite AKARI
}

\author{
Fumihiko Usui $^{1}$, Toshihiro Kasuga ${ }^{2}$, Sunao Hasegawa ${ }^{1}$, Masateru Ishiguro ${ }^{3}$, Daisuke \\ Kuroda $^{4}$, Thomas G. Müller ${ }^{5}$, Takafumi Ootsubo ${ }^{6}$, and Hideo Matsuhara ${ }^{1}$ \\ usui@ir.isas.jaxa.jp
}

\begin{abstract}
We present an analysis of the albedo properties of main belt asteroids detected by the All-Sky Survey of the infrared astronomical satellite AKARI. The characteristics of 5120 asteroids detected by the survey, including their sizes and albedos, were cataloged in the Asteroid Catalog Using AKARI (AcuA). Size and albedo measurements were based on the Standard Thermal Model, using inputs of infrared fluxes and absolute magnitudes measured at optical wavelengths. Main belt asteroids, which account for 4722 of the 5120 AcuA asteroids, have semimajor axes of 2.06 to $3.27 \mathrm{AU}$, except for the near-Earth asteroids. AcuA provides a complete data set of all main belt asteroids brighter than the absolute magnitude of $H<10.3$, which corresponds to the diameter of $d>20 \mathrm{~km}$. We confirmed that the albedo distribution of the main belt asteroids is strongly bimodal as was already known from the past observations, and that the bimodal distribution occurs not only in the total population, but also within inner, middle, and outer regions of the main belt. The bimodal distribution in each group consists of low-albedo components in C-type asteroids and high albedo components in S-type asteroids. We found that the small asteroids have much more variety in
\end{abstract}

\footnotetext{
${ }^{1}$ Institute of Space and Astronautical Science, Japan Aerospace Exploration Agency, 3-1-1 Yoshinodai, Chuo-ku, Sagamihara 252-5210, Japan

${ }^{2}$ Public Relations Center, National Astronomical Observatory of Japan, 2-21-1 Osawa, Mitaka, Tokyo 181-8588, Japan

${ }^{3}$ Department of Physics and Astronomy, Seoul National University, San 56-1, Shillim-dong Gwanak-gu, Seoul 151-742, South Korea

${ }^{4}$ Okayama Astrophysical Observatory, National Astronomical Observatory, 3037-5 Honjo, Kamogata-cho, Asakuchi, Okayama 719-0232, Japan

${ }^{5}$ Max-Planck-Institut für Extraterrestrische Physik, Giessenbachstraße, 85748 Garching, Germany

${ }^{6}$ Astronomical Institute, Tohoku University, 6-3 Aoba, Aramaki, Aoba-ku, Sendai 980-8578, Japan
} 
albedo than the large asteroids. In spite of the albedo transition process like space weathering, the heliocentric distribution of the mean albedo of asteroids in each taxonomic type is nearly flat. The mean albedo of the total, on the other hand, gradually decreases with an increase in semimajor axis. This can be explained by the compositional ratio of taxonomic types; that is, the proportion of dark asteroids such as C- and D-types increases, while that of bright asteroids such as S-type decreases, with increasing heliocentric distance. The heliocentric distributions of X-subclasses: E-, M-, and P-type, which can be divided based on albedo values, are also examined. P-type, which is the major component in $\mathrm{X}$-types, are distributed throughout the main belt regions, and the abundance of P-type increases beyond $3 \mathrm{AU}$. This distribution is similar to that of C- or D-types.

Subject headings: catalogs — infrared: planetary systems - minor planets, asteroids: general — space vehicles — surveys

\section{Introduction}

Studies of the physical properties of asteroids are of fundamental importance to our understanding of the origin, evolution, and structure of the solar system. In order to investigate the compositions of asteroids and the chemical and thermal processes of alternations, it is essential to examine detailed mineralogical characterizations of individual asteroids (e.g., Gaffey et al. (2002)). Taxonomic classifications of asteroids represent a broader, alternative approach for appraising the compositions and surface conditions for large numbers of asteroids. As is commonly accepted (and first explicitly described by Chapman et al. (1975)), most asteroids can be classified as either C- or S-type. C-type asteroids are historically associated with carbonaceous chondrites, and S-type asteroids with stony-iron meteorites.

Several outstanding works have defined methodologies for the taxonomic classification

of asteroids (e.g., Tholen (1984); Bus (1999); Lazzaro et al. (2004); Carvano et al. (2010)). These taxonomies are based on statistically processed information parameterizing groundbased spectroscopic observations within optical and near-infrared wavelengths. One of the notable results of these taxonomic classifications, as presented by Bus \& Binzel (2002) (and also by Mothé-Diniz et al. (2003)) is the non-uniform heliocentric clustering of taxonomic types in main belt regions; asteroids of each taxonomic type $(\mathrm{C}, \mathrm{S}, \mathrm{X}, \mathrm{D}+\mathrm{Ld}+\mathrm{T}$, and V) show bias-corrected distributions at heliocentric distances of 2.1 to 3.3 AU. The interpretation of these results is that S-, C-, and D-type asteroids are distributed progressively further from 
the sun in that order, and that the bodies located further from the sun are less affected by metamorphism and contain a greater proportion of primitive materials than those closer to the sun (i.e., S-type asteroids are the most metamorphosed and contain the least amount of primitive material and D-type asteroids are the least metamorphosed and contain the greatest proportion of volatile material).

Albedo data, along with taxonomic classifications of asteroids, also contribute to our understanding of the large-scale distribution of asteroid compositions. Albedo values are strongly dependent on the surface conditions and compositions of asteroids. The relationship between taxonomic types and albedo is, on the other hand, complex, and type determinations cannot be made on the basis of albedo values alone; the albedos of C- and S-type asteroids vary widely, even though the albedo of C-types is generally low and the albedo of S-types is generally high (e.g., Zellner \& Gradie (1976)).

The most direct way to measure the albedo of asteroids is by in-situ observations using flyby and rendezvous missions (e.g., the spacecrafts Galileo (Johnson et al. 1992) for (951) Gaspra in 1991 and (243) Ida/Dactyl in 1993; NEAR Shoemaker (Cheng et al. 1997) for (253) Mathilde in 1996 and (433) Eros in 2000; Deep Space 1 (Ravman 2003) for (9969) Braille in 1999; Stardust-NExT (Brownlee et al. 2003) for (5535) Annefrank in 2002; New Horizons (Stern \& Spencer 2003) for (132524) APL in 2006; Rosetta (Glassmeier et al. 2007) for (2867) Steins in 2008 and (21) Lutetia in 2010; Dawn (Russell et al. 2004) for (4) Vesta in 2011-2012). Moreover, the sample return from asteroids (by Hayabusa from (25143) Itokawa in 2005; Fujiwara et al. (2006)) can provide materials for analytical laboratory determinations of asteroid compositions. The two most important physical characteristics of asteroids, albedo and size, are coupled by their relationships with the absolute magnitude (e.g., Fowler \& Chillemi (1992)); given the size of an asteroid, the albedo value can be uniquely derived on the basis of its absolute magnitude.

Several imaging techniques have been developed to determine the shape of asteroids. The most straightforward approach is by direct imaging of the asteroid, for example, by use of the Hubble Space Telescope (Li et al. 2010) or large ground-based telescopes with adaptive optics (Drummond et al. 2009). Radar observations (Ostro et al. 2000) and speckle interferometry (Drummond et al. 1985) are also useful for resolving the shapes of asteroids, as are stellar occultations (Durech et al. 2011); the latter is a powerful approach, which relies upon well-organized campaign observations by many participants including amateur astronomers. Although such methods are readily available, they require the convergence of critical conditions, such as the selection of large targets with trajectories approaching the earth, and/or narrow observational windows. The sheer number of asteroids poses yet another difficulty; as of 2012, the number of known asteroids is more than 595,000 (see 
e.g., Minor Planet Center 1 at the Smithsonian Astrophysical Observatory), a number which precludes detailed observations of all individual bodies.

One of the most effective indirect methods for measuring the sizes and albedos of asteroids is by radiometry, which uses the combined flux measurements at thermal infrared and reflected optical wavelengths. The radiometric technique for determining the sizes and albedos of asteroids was developed in the early 1970s using ground-based observatories (Allen 1970, 1971; Matson 1971), and the approach has yielded a wealth of information both on individual objects and large populations of asteroids. Using radiometric measurements, a large number of objects can be observed in a short period of time, thus providing uniform data for large, relatively unbiased populations within the asteroid belt. Although radiometry requires careful calibration, once calibrated, this method can obtain "wholesale" highly accurate measurements of the physical properties of large numbers of asteroids.

Infrared observations using ground-based observatories, especially in the mid-infrared range, are severely limited on account of moisture conditions in the telluric atmosphere; however, infrared measurements obtained using space-borne telescopes are very reliable. Furthermore, when integrated into an all-sky survey, large numbers of infrared images can be obtained rapidly; moreover, the data are unbiased and uniform. The first systematic survey of asteroids using a space telescope was made by the Infrared Astronomical Satellite (IRAS; Neugebauer et al. (1984)), which cataloged the sizes and albedos of 2470 asteroids (Tedesco et al. 2002). Recently, the asteroid catalog was updated with data from the infrared astronomical satellite AKARI All-Sky Survey (Murakami et al. 2007). The Asteroid Catalog Using AKARI (AcuA) contains an inventory of the sizes and albedos of 5120 asteroids (Usui et al. 2011). The Wide-field Infrared Survey Explorer (WISE; Wright et al. (2010)) also performed an infrared all-sky survey and the processed data including large amount of asteroid information (WISE/NEOWISE; Mainzer et al. (2011)) are being compiled.

This paper focuses on the albedos of the main belt asteroids (MBAs) measured by $A K A R I$. The spatial distribution of compositions among MBAs is of particular interest, because the main belt is the largest reservoir of asteroids in the solar system; more than $90 \%$ of asteroids with known orbital elements are classified as MBAs. Asteroids are thought to be the remnants of planetesimals formed in the early solar system (Bottke et al. 2002a). Some were formed near to their current locations, and others have migrated from their original birthplaces in conjunction with the migrations of giant planets (e.g., Levison et al. (2009)). Because of cataclysmic events in the history of the solar system, present-day asteroids, especially MBAs, are well mixed and represent multiple origins; they are confined to certain

\footnotetext{
${ }^{1}$ http://www.minorplanetcenter.net
} 
regions on account of resonance effects, and/or have been broken or segmented by mutual collisions. Some asteroids originally formed in volatile-poor regions (i.e., the inner parts of the solar system), whereas others formed in volatile-rich regions (i.e., the outer parts of the solar system, beyond the snow line at the times of formation). Processes of dynamical evolution and chemical processing may have affected the physical conditions, and hence the albedo properties, of asteroid surfaces.

The main purpose of this study is to examine the size dependencies, frequency distributions, and heliocentric distributions of the albedos of AcuA MBAs, by comparing to recent taxonomic classifications. Famed results about the heliocentric distribution of taxonomic types are presented in Bus \& Binzel (2002) and Mothé-Diniz et al. (2003). They performed

a bias correction method based on Zellner (1979) and Bus (1999), that is, asteroids are divided into several zones according to the semimajor axis and the absolute magnitude, and the ratio between the total number of asteroids and the number of classified asteroids in each zone are determined, then this ratio is used as the bias correction factor for estimating the fraction of taxonomies of unclassified objects under the assumption of size and albedo based on the IRAS data set. AcuA, which is based on an all-sky survey that lasted more than one year, provides a complete data set of all known asteroids brighter than the absolute magnitude of $H<10.3$ for MBAs, which correspond to the size for $d>20 \mathrm{~km}$. When the objects larger than $20 \mathrm{~km}$ are used for analysis, no assumptions about size or albedo are needed. Thus we have carried out no bias correction for the magnitude incompleteness in our examination of the albedo properties of MBAs. The paper is organized as follows. In Section 2, we describe the features of the AcuA data base and the division of the main belt into three regions: inner, middle, and outer. In Section 3, we describe investigations of albedo properties of MBAs in the context of taxonomic classification. In Section 4, we discuss the reasons for the albedo dependencies which are described in Section 3. Finally, Section 5 provides a summary.

\section{AcuA main belt asteroids}

\subsection{The AcuA archival data base}

AKARI (Murakami et al. 2007) surveyed more than $96 \%$ of the sky during the 18 month course of the cryogenic cooled phase from 2006 to 2007. Data were obtained for 6 bands in the mid- to far-infrared spectral range, with a solar elongation of $90^{\circ} \pm 1^{\circ}$. The mid-infrared part of the All-Sky Survey was conducted at two broad bands centered at 9 $\mu \mathrm{m}(S 9 W)$ and $18 \mu \mathrm{m}(L 18 W)$, using an on-board InfraRed Camera (IRC; Onaka et al. (2007)). The $5 \sigma$ detection limits at $S 9 W$ and $L 18 W$ were 50 and $90 \mathrm{mJy}$, respectively, and 
the spatial resolution of the IRC in the All-Sky Survey mode was less than $\sim 10^{\prime \prime}$ per pixel (Ishihara et al. 2010). Point sources were extracted from the IRC All-Sky Survey image data, numbering 4.8 million at $S 9 \mathrm{~W}$ and 1.2 million at $L 18 \mathrm{~W}$. The sources which are detected more than twice are compiled in the IRC Point Source Catalog (IRC-PSC; Ishihara et al. (2010)); however, about $20 \%$ of the extracted sources were not contributed for IRC-PSC and were remained unused because of a lack of multiple detection at the same position of the sky. Asteroids were included in these "residual" sources, due to their motions against the background sky. By matching the data with the positions of known asteroids, about 7000 point sources at $S 9 \mathrm{~W}$ and 14000 point sources at $L 18 \mathrm{~W}$ were identified as asteroids, corresponding to 5120 objects without duplications.

The radiometric diameters $(d)$ and geometric albedos in the visible wavelengths $\left(p_{\mathrm{v}}\right)$ of the identified asteroids were calculated using the Standard Thermal Model (Lebofsky et al. 1986) with adjusted beaming parameters of $\eta=0.87$ for $S 9 W$ and 0.77 for $L 18 W$ to reduce the total systematic errors. Taking into account several uncertainties, such as the values of the absolute magnitude and the phase slope parameter, we computed typical uncertainties in the diameters of asteroids to be about $4.7 \%$, and those in the geometric albedo results to be about $10.1 \%$. The AKARI asteroid catalog developed on the basis of these analyses contains data on the sizes and albedos of 5120 asteroids. The catalog is named AcuA 2 , which is the acronym for Asteroid Catalog Using AKARI. More details are described in Usui et al. (2011).

Figure 1 shows the distribution of $H$ for the AcuA asteroids. This figure can be interpreted as reflecting the completeness of detections of known asteroids with size and albedo data. AcuA, which was constructed based on 16 months of all-sky survey data, provides a complete data set of all asteroids brighter than absolute magnitude of $H<9$ within the semimajor axis of $a<6 \mathrm{AU}$, and $H<10.3$ for all MBAs. $H<10.3$ for MBAs corresponds to $d>20 \mathrm{~km}$ in size. Thus, all AcuA MBAs with values of $d>20 \mathrm{~km}$ (a total of 1974) are mainly used in the following discussion.

Based on more recent studies, the uncertainties of the thermal model calculation in Usui et al. (2011) can be reevaluated. Pravec et al. (2012) calculated the absolute magnitudes for 583 asteroids from their dedicated photometric observations over thirty years. When compared with the existing database, the difference in absolute magnitude between the database values and their new estimation is about 0.08 within the range of $H<10.3$. The divergence of the slope G parameters are also given in Pravec et al. (2012) as about 0.083 , though this has little influence on the infrared fluxes, at less than one percent. The

\footnotetext{
${ }^{2}$ The data are available at http://darts.jaxa.jp/ir/akari/catalogue/AcuA.html
} 
variation of the beaming parameter is given as 0.157 from Masiero et al. (2011). These lead to new estimates in the typical uncertainties of AcuA in the diameters to $13.6 \%$, and those in the albedo to $28.1 \%$.

\subsection{Divisions of the main belt asteroids}

The number of asteroids inventoried in the AcuA is summarized in Table1. Determinations of each taxonomic type are based on Tholen (1984); Bus (1999); Lazzaro et al. (2004); Carvano et al. (2010), and other references summarized in Usui et al. (2011), appendix 4. Figure 2 shows histograms of the numbers of AcuA asteroids as a function of the semimajor axes. We focus on three regions of the main belt which are defined on the basis of semimajor axis: the inner $(2.06<a \leq 2.50)$, middle $(2.50<a \leq 2.82)$, and outer $(2.82<a \leq 3.27)$ regions, where $a$ is the semimajor axis in AUs. It should be noted that some near-Earth asteroids (Apollos and Amors) which occasionally have semimajor axis in the ranges of MBAs, are not considered in the following discussion, and the other group of near-Earth asteroids in which $a<1 \mathrm{AU}$ (Atens) are, by definition, not within main belt regions.

The orbital elements of the asteroids were obtained from the Asteroid Orbital Elements Database (Bowell et al. 1994) distributed by Lowell Observatory 3 . The boundaries of the main belt regions at the semimajor axis $a=2.06,2.50,2.82$, and 3.27 AU correspond, respectively, to the 4:1, 3:1, 5:2, and 2:1 mean motion resonances of Jupiter (e.g., Kirkwood (1867); Froeschle \& Greenberg (1989); Scholl et al. (1989); Yoshikawa (1989)). The mean motions and secular resonances of Jupiter are the dominant effects on the dynamical evolution of MBAs at the present stage of solar system evolution. The Yarkovsky thermal force (Bottke et al. 2002b) is also known to cause changes in the orbits of asteroids (mainly affecting the semimajor axis), but its effects are less than those of the dynamical resonances on large-size objects. The numbers of AcuA asteroids in each region of the main belt (inner, middle, and outer) is 858, 1523, and 2341, respectively (also see Table 1). The number of detected asteroids in the outer region is greater than that in the inner region, implying that the actual number of asteroids is greater in outer regions, compared to the number of observed asteroids, which generally decreases as a function of distance on account of distance-dependent instrumental detection limits; however, the precise number density of asteroids in each region was not examined. Moreover, the number of inner region MBAs is about half that of middle region MBAs, despite the fact that inner region MBAs are more readily detected. Figure 3 also illustrates the size distribution of the inner, middle, and outer

\footnotetext{
${ }^{3}$ The data are available at ftp://ftp.lowell.edu/pub/elgb/astorb.html
} 
MBAs. Concerning the size distribution of asteroids, the number of asteroids is expected to increase monotonically with decrease in size. This figure, however, shows maxima at $d \sim 20 \mathrm{~km}$ for the outer MBAs. This suggests that the survey completeness rapidly drops for asteroids smaller than $\sim 20 \mathrm{~km}$ in the outer region on account of the detection limit of $A K A R I$ instrumentation. This size limit is consistent with the fact that AcuA is complete for all MBAs with $H<10.3$, as described in Section 2.1. In this paper, we consider only the inner, middle, and outer MBAs in our comprehensive analysis of the asteroid belt; we do not consider objects outside of the main belt $(a \leq 2.06$, or $a>3.27)$ and do not investigate the properties of the individual dynamical families in detail, which will be discussed in separate papers; e.g., the Cybele family is studied in Kasuga et al. (2012).

\section{Albedo properties of MBAs}

\subsection{Albedo size-dependencies}

Figure 4 shows the distributions of albedo values as a function of asteroid diameter $(d)$, for asteroids cataloged in the AcuA. The total number of asteroids with $d<5 \mathrm{~km}$ is relatively low, on account of the detection limit for small bodies (see Usui et al. (2011), Figure 8a). Notably, the distributions of albedo values for the asteroids in each region are bimodal.

Figure [5 shows the distribution of albedo values of AcuA MBAs as a function of asteroid size, separated by taxonomic type. It should be noted that the taxonomic type is known for 2496 AcuA asteroids (53\% of the total number of detected MBAs). It should also be noted that the taxonomic type was determined using data from the literature mentioned above, and not from $A K A R I$ observations; this is because (1) the $A K A R I$ observations were conducted at infrared, not optical, wavelengths, and (2) the two $A K A R I$ mid-infrared channels $(S 9 W$ and $L 18 W$ ) did not observe the same region of the sky simultaneously; thus, a complete data set for each asteroid was not always obtained (see Usui et al. (2011), details). Figure 5 reveals that the clusters of high- and low-albedo asteroids depicted in Figure 4 correspond to S-type and C-types asteroids. In both distributions, it is found that the scatter in albedo among the smaller asteroids increases. The albedo values of X-type asteroids are widely distributed. The albedo values of D-type asteroids are moderately low, and the number of D-type asteroids is relatively small (see Table 2).

X-type asteroids, which have featureless flat or slightly reddened spectra over optical wavelengths, are spectrally degenerate and can be classified into three subclasses only on the basis of albedo values (Tholen \& Barucci 1989; Clark et al. 2004; Fornasier et al. 2011), 
as E-type: $p_{\mathrm{v}}>0.3$ (high albedo), M-type: $0.3 \geq p_{\mathrm{v}}>0.1$ (medium albedo), and P-type: $0.1 \geq p_{\mathrm{v}}$ (low albedo). These three classes are spectrally similar to each other, but have very different inferred mineralogy, as E-type: containing enstatite-rich aubrites, M-type: containing metallic iron cores, and P-type: carbon and/or organic-rich. According to the classification based on albedo values, of the 490 X-type AcuA MBAs, 14 are E-type, 145 are M-type, and 331 are P-type. Note that for $90 \%$ of these X-type MBAs, the AcuA albedos provide the first sub-classification into E-, M-, and P-types. The largest E-type member is (71) Niobe $\left(d=80.86 \mathrm{~km}, p_{\mathrm{v}}=0.326\right)$ in the middle MBAs. The mean albedos of each subclass are presented in Table 3. Note that there are two objects that show inconsistency between taxonomic type and albedo value. While (498) Tokio was classified as an M-type asteroid in a previous work (Tholen 1984), the albedo value of this object is $p_{\mathrm{v}}=0.063$ by $A K A R I$ (or 0.069 by the IRAS); thus, (498) is a P-type, not an M-type, asteroid. (55) Pandora was also classified as an M-type asteroid (also by Tholen (1984)); however, it is an E-type asteroid $\left(p_{\mathrm{v}}=0.337\right.$ by $A K A R I$ or 0.301 by the $\left.I R A S\right)$. (498) and (55) are middle MBAs. Figure 6 shows the albedo distribution of X-type asteroids. There are two major components found in this figure, the M-type with $p_{\mathrm{v}}>0.1$ and P-type with $p_{\mathrm{v}} \leq 0.1$. From this distribution, it seems reasonable that the boundary between medium-albedo M-type and low-albedo P-type is set as $p_{\mathrm{v}}=0.1$. On the other hand, no clear boundary is found around $p_{\mathrm{v}} \sim 0.3$, between E- and M-type.

Two large and high-albedo asteroids are observed among the D-types: (9) Metis $(d=166$ $\left.\mathrm{km}, p_{\mathrm{v}}=0.213\right)$ is an inner MBA, and (224) Oceana $\left(d=54 \mathrm{~km}, p_{\mathrm{v}}=0.222\right)$ is a middle MBA. These were considered as D-type asteroids by Lazzaro et al. (2004), but were recently reclassified as S-type (9) and M-type (224) asteroids (Neese 2010); albedo values for these asteroids in the AcuA also support this classification of Neese (2010).

\subsection{Variations in the distributions of albedo values}

Figure 7 shows histograms of asteroid albedo values for the inner, middle, and outer region. In Figure 4 (also see Usui et al. (2011), Figure 8b), the "bimodal" distribution of albedo is clearly visible. The population densities of the low- and high-albedo components are, however, not the same; the peak of the low albedo distribution is about twice that of the high albedo distribution. This same pattern is observed in the inner, middle, and outer region distributions as Figure 4, but the detailed features of each distribution vary. In the inner region (red lines in Figure 7), the peak of the high albedo distribution is nearly the same as, or a little higher than, that of the lower albedo distribution. In the middle and outer region asteroids (green and blue lines in Figure 7), the low albedo component is 
dominant, and the peak of the high albedo distribution is nearly buried in the long tail of the low albedo component; this pattern is especially prominent in the albedo distributions of the smaller asteroids.

Another outstanding feature of the albedo distributions is that, for the large asteroids (Figure $7 \mathrm{k}$ ), the distributions are clearly divided at $p_{\mathrm{v}} \sim 0.1$; this division is less pronounced in the small asteroids; the mean albedo value of the total population of 4722 AcuA MBAs is $\overline{p_{\mathrm{v}}}=0.102 \pm 0.079$. This division in the albedo distribution was also observed in data from the IRAS asteroid catalog (Tedesco et al. 2002); however, the boundary was observed at $p_{\mathrm{v}}=0.089$ (Morbidelli et al. 2002). Similar features are found in the WISE/NEOWISE data set (Masiero et al. 2011).

Figure 8 shows the heliocentric distribution for asteroids with diameters $d>20 \mathrm{~km}$ for each of the taxonomic types. AcuA covers a complete data set of all MBAs with $d>20 \mathrm{~km}$. We should note that there is a possible selection effect in the determination of the taxonomic type, i.e., spectroscopic data have been preferentially obtained from brighter (larger size, higher albedo) objects and/or particular family members, and hence these objects are more heavily represented in the data than are darker objects.

Figure $8 \mathrm{a}$ illustrates the numbers of AcuA MBAs of each taxonomic type. As observed in Figure 2, the number of detected asteroids increases with increasing semimajor axis, except for large deviations at $a \sim 2.8$ AU. Taxonomic classifications were determined for 1409 asteroids (71\% of the AcuA MBAs with sizes $d>20 \mathrm{~km}$ ), representing the following regional distribution: 144 inner, 463 middle, and 802 outer MBAs. Percentage of asteroids with taxonomy determinations in each region is $92 \%, 88 \%$, and $62 \%$, respectively. Fewer classifications exist in outer region due to the selection effect as mentioned above. Figure 8b shows the fractional representation of each taxonomic type at different semimajor axis, as well as the results obtained by Bus \& Binzel (2002) (dashed lines). Bus \& Binzel (2002) showed the bias-corrected heliocentric distribution for each taxonomic type in 1447 asteroids of size $d>20 \mathrm{~km}$, which is the same size range in this work. We observed distributions of S-type and D-type asteroids similar to those of Bus \& Binzel (2002); that is, S-type asteroids are dominant in the inner region, with relative proportions decreasing with an increase in semimajor axis; D-type asteroids comprise $<10 \%$ of the total number of asteroids, but their relative proportions gradually increase with increasing semimajor axis. Our data on the fractions of C-type and X-type asteroids depart from those of Bus \& Binzel (2002) by $\sim 10 \%$; the fraction of C-type in this work is less than that in Bus \& Binzel (2002), and that of X-type is more than that in Bus \& Binzel (2002). This result could be caused by biases related to the number of taxonomic identifications. In the literature, X-types crowd around 3 AU (Mothé-Diniz et al. 2003) and the Hungaria family (Warner et al. 2009). The 
former group can be confirmed from our sample in Figure 8b. The latter is in the range of $1.78<a<2.06 \mathrm{AU}$ (Gradie et al. 1979) and out of the MBAs. The number of AcuA V-type asteroids is small ((4) Vesta, (854) Frostia, (1273) Helma, (1981) Midas, and (3657) Ermolova; (1981) is a near-Earth asteroid (Apollos), not a MBA, and the other four are inner MBAs), and (4) is the only asteroid larger than $20 \mathrm{~km}$. These V-type asteroids are excluded from this study.

Figure 9 shows the heliocentric distribution for subclasses of X-types from AcuA asteroids with diameters $d>20 \mathrm{~km}$ : E-, M-, and P-type. Traditionally the distribution of X-subclasses were described in Bell et al. (1989) based on Tholen taxonomy (Tholen 1984), however they did not have detailed albedo information. Figure 9 shows revised distributions based on the AcuA data set. The number of identified E-type asteroids larger than $20 \mathrm{~km}$ is only seven (see, Table 4). These large E-type asteroids are non-family members except for (44) Nysa. Although E-type members are primarily located in the inner asteroid belt, five of seven large E-types are the middle MBAs. One E-type in the outer MBAs is (665) Sabine $\left(d=53.01 \mathrm{~km}, p_{\mathrm{v}}=0.365, a=3.14 \mathrm{AU}\right)$, which has a detailed shape model (Michałowski et al. 2006). A major group in the X-subclasses is the P-type, which accounts for $68 \%$ of our X-type sample $(d>20 \mathrm{~km})$. Throughout the main belt region, P-type asteroids are dominant among the X-types. In Figure 9b, the abundance of P-types increases beyond $3 \mathrm{AU}$, while that of M-types decreases. The distribution of P-types is similar to that of C- or D-types in Figure 8 $\mathrm{b}$. The mean albedo of P-types is $0.054 \pm 0.017$ of total 251 (Table 3), which is also similar to C: $0.066 \pm 0.031$, or D: $0.077 \pm 0.041$ (Table 2). From this, it can be conjectured that P-type asteroids have similar origin or similar evolutional process, to C- or D-types, though the actual component of X-types are not fully understood yet.

Figure 10 presents the dependency of mean albedo on heliocentric distance. In the total population of asteroids, albedo gradually decreases as the semimajor axis increases; i.e., asteroids located further from the sun are darker. On the other hand, within individual taxonomic types, the heliocentric distributions of mean albedo are nearly constant throughout the entire main belt, although albedo variations at any given distance are large. Thus, the albedo within a given taxonomic type is relatively independent of heliocentric distance. The cause of the decline in the mean albedo of the total distribution in outer regions is not the heliocentric distance itself, but the influence of the compositional mixing ratios of taxonomic types, as observed in Figure 8 . 


\section{Discussion}

As observed in Figure 5, the albedo of asteroids is dependent on its size. This does not mean, however, that smaller asteroids tend to have higher albedos; rather, it implies that the diversity of albedo values is greater for smaller asteroids than for larger asteroids. AcuA provides a complete data set for $H<10.3$, which corresponds to $d>20 \mathrm{~km}$. Moreover, detectability with $A K A R I$ does not depend on albedo. This is because the thermal flux of an asteroid is hardly dependent on albedo (surface temperature is proportional to ( 1 $\left.A_{\mathrm{B}}\right)^{1 / 4}$, which has a value in a range from 0.99 to 0.93 with $p_{\mathrm{v}}$ of 0.01 to 0.6 , where $A_{\mathrm{B}}=$ $(0.290+0.684 G) p_{\mathrm{v}}$ is the Bond albedo), while the visible reflected component of sunlight is proportional to the albedo. For these reasons, the scatter in albedo among the smaller objects should be real, not a bias effect; even so, taxonomic information is available only for $53 \%$ of the total MBAs in AcuA, or $71 \%$ of $d>20 \mathrm{~km}$ MBAs in AcuA. Taxonomic classifications are derived from spectroscopic studies of asteroids, which have strong observational selection biases. Especially for asteroid with $d<20 \mathrm{~km}$, our study of the size-albedo distribution of MBAs rapidly becomes incomplete due to the sensitivity limits of $A K A R I$.

Spacecraft explorations show that most asteroid surfaces are covered with regolith and/or numerous boulders in a wide range of sizes. For example, a unique boulder with an unusually low brightness is found to sit on top of the asteroid (25143) Itokawa like a benchmark (Fujiwara et al. 2006). Several interpretations for the origin of this distinct "black boulder", which is about $40 \%$ darker than surrounding materials, have been considered (e.g., Hirata \& Ishiguro (2011)). Such local heterogeneities are averaged out and nearly homogenized in the global view, and only a small degree of regional variation on surfaces is observed. However, especially for smaller asteroids, the effects of these heterogeneities are conspicuous relative to their sizes. This could partly explain the albedo variations at smaller sizes.

For S-type asteroids, albedo dependency can be explained by the space weathering of stony chondritic asteroids. The collisional life times and surface ages of S-type asteroids are correlated with their sizes (Davis et al. 2002; Bottke et al. 2005; Nesvorný et al. 2005); thus, fresher and smaller objects are less space weathered and their albedos are relatively higher (Burbine et al. 2008; Thomas et al. 2011). Space weathering effects cause the visible and near-infrared reflectance spectra of stony asteroids to be darker and redder than those of pristine materials (Sasaki et al. 2001). Ground-based observations suggest that a color transition exists between ordinary chondrite-like objects and S-type asteroids over the size range $0.1-5 \mathrm{~km}$, which implies that the surfaces of small stony asteroids are evolving towards

the colors of large asteroids (Binzel et al. 2004, 2010). Our data indicate that the albedo transition occurs in the size range of $d>5 \mathrm{~km}$. 
In contrast to S-type asteroids, the reason for the albedo transition in C-type asteroids is less known. The relationship between age and the space weathering effect in C-type asteroids has been mentioned in several papers (e.g., Nesvorný et al. (2005); Lazzarin et al. (2006) ), however, the relationship is still uncertain, mainly on account of the "darkness" of C-type asteroids. Laboratory measurements indicate that thermally metamorphosed samples of carbonaceous chondrites possess high absolute reflectance (Hiroi et al. 1994), which could cause the high albedo of asteroids. Figure 11 shows the dependence of the absolute reflectance of chondrites on the degree of metamorphism (Clark et al. 2009). The classification of metamorphism in chondritic meteorites is qualitatively determined (Weisberg et al. 2006): the most pristine materials are type 3; types 4 to 6 represent an increasing degree of thermal metamorphism, and types 2 to 1 represent an increasing degree of aqueous alteration. While thermal metamorphism and aqueous alteration are related to different mechanisms of recrystallization, the classification from 1 to 6 can be taken to represent an increasing degree of metamorphism. The metamorphism of chondrites occurs in the inner parts of the parent body, which is internally heated by the decay energy of short-lived radioisotopes (e.g., Trieloff et al. (2003); Greenwood et al. (2010)). In a catastrophic disruption caused by a highly energetic impact, small fragments, including those from the inner portion of a parent body, are exposed. Asteroids smaller than a few 10s of kilometers are considered to be formed mainly by collisional breakup (Bottke et al. 2005); thus, the high albedo of some materials may have been derived from deeper thermal metamorphic processes. In other materials, however, the albedo might be due to the presence of reaccumulated fragments (e.g., Davis et al. (1979); Fujiwara (1982)), and/or a regolith layer (Hiroi \& Pieters 1992, 1994). Thus, a variety of processes might cause the albedo variation at smaller sizes.

No clear dependency of albedo on size is found for D-type asteroids in Figure 5d, while the number of detected D-types is relatively small. Some researchers have distinguished between the features of inner D-type and outer D-type varieties of D-type asteroids (e.g., Lagerkvist et al. (2005); Mothé-Diniz (2009)). D-type asteroids dominate the Jovian Trojans, but become rarer at smaller heliocentric distances. As discussed by Carvano et al. (2003), the variations in D-type asteroids might be related to different origins and evolutionary histories, or to different processes of differentiation occurring closer to the sun. Indeed, the actual mineralogical characterization of D-type asteroids is difficult to ascertain because of their small number of samples (except for the Tagish Lake meteorite; Hiroi et al. (2001)). 


\section{Summary}

We have presented an analysis of the albedo properties of MBAs detected by $A K A R I$ and recorded in the AcuA. Of the 5120 AcuA asteroids, 4722 are MBAs, and 1974 are provided as a complete data set of MBAs larger than $d>20 \mathrm{~km}$. As is already known, the albedo distribution of MBAs is strongly bimodal; this trend is present not only in the distribution of the total population, but also in the distributions of inner, middle, and outer MBAs. The bimodal distributions are separated into two major groups at an albedo value of $p_{\mathrm{v}}=0.1$, which demarcates low-albedo C-type and high-albedo S-type asteroids. In each group, the albedo distribution is size-dependent, and the variation in albedo values is greater at smaller sizes. For smaller asteroids, the effects of surface heterogeneities on albedo are relatively large, while such local heterogeneities are averaged out and seemingly homogenized for larger asteroids. Moreover, albedo distributions in S-type asteroids appear to be affected by the space weathering, whereas the albedo distributions in C-type asteroids are partially explained by the effects of metamorphism.

We examined the heliocentric distributions of mean albedo values for each taxonomic type. In spite of the influence of the space weathering and other albedo transition processes, the mean albedo is nearly constant and independent of heliocentric distance throughout the entire main belt region, irrespective of taxonomic type. In the total distribution, on the other hand, the mean albedo value gradually decreases with increasing the semimajor axis, presumably due to the compositional mixing ratios of taxonomic types.

Almost $90 \%$ of the X-type MBAs in the AcuA data set can now be subdivided into the E-, M-, and P-types based on the AKARI-derived albedos. The distribution of P-types, which have lower albedos among the X-types, are spread throughout the main belt regions, and increases beyond 3 AU, while the proportion of medium-albedo M-types decreases. Ptype asteroids are considered to have similar origin or similar evolutional process, to C- or D-types.

This study is based on observations with $A K A R I$, a JAXA project with the participation of ESA. We would like to thank all members of the $A K A R I$ project for their dedicated efforts to generate this data base. This study was supported in part by ISAS/JAXA as a collaborative program with the Space Plasma Experiment. This work is supported in part by a Grant-in-Aid for Scientific Research on Priority Areas No. 19047003 to DK, and Grantsin-Aid for Young Scientists (B) No. 21740153 and Scientific Research on Innovative Areas No. 21111005 to TO. Finally, we are very grateful to the anonymous reviewer, for her/his constructive comments. 


\section{A. Mean albedo for each taxonomic class}

Tables 5, 6, and 7 summarize the mean albedo of each taxonomic class, classified according to Tholen taxonomy (Tholen 1984; Lazzaro et al. 2004), Bus taxonomy (Bus 1999; Lazzaro et al. 2004), and Carvano taxonomy (Carvano et al. 2010), respectively. 
Table 1. Numbers of asteroids detected by $A K A R I$, classified by dynamical group and by main belt region.

\begin{tabular}{|c|c|c|c|c|c|c|c|}
\hline Dynamical group & $\mathrm{C}$ & $\mathrm{S}$ & $\mathrm{X}$ & $\mathrm{D}$ & $\mathrm{V}$ & Unclassified & Total \\
\hline Near-Earth Asteroids & 2 & 16 & 2 & - & 1 & 37 & 58 \\
\hline Main Belt Asteroids & 1150 & 722 & 490 & 130 & 4 & 2226 & 4722 \\
\hline Cybeles & 22 & 2 & 26 & 26 & - & 29 & 105 \\
\hline Hildas & 1 & 1 & 19 & 30 & - & 35 & 86 \\
\hline Jovian Trojans (L4, L5) & 6 & - & 7 & 53 & - & 43 & 109 \\
\hline Others $^{\mathrm{a}}$ & 2 & 17 & 6 & 2 & - & 13 & 40 \\
\hline Total & 1183 & 758 & 550 & 241 & 5 & 2383 & 5120 \\
\hline \multicolumn{8}{|l|}{ MBAs subtotal } \\
\hline Inner & 160 & 242 & 64 & 16 & 4 & 372 & 858 \\
\hline Middle & 401 & 267 & 168 & 28 & - & 659 & 1523 \\
\hline Outer & 589 & 213 & 258 & 86 & - & 1195 & 2341 \\
\hline Total & 1150 & 722 & 490 & 130 & 4 & 2226 & 4722 \\
\hline \multicolumn{8}{|c|}{ MBAs $(d>20 \mathrm{~km})$ subtotal } \\
\hline Inner & 48 & 56 & 32 & 8 & 1 & 11 & 156 \\
\hline Middle & 184 & 138 & 124 & 17 & - & 61 & 524 \\
\hline Outer & 381 & 147 & 212 & 62 & - & 492 & 1294 \\
\hline Total & 613 & 341 & 368 & 87 & 1 & 564 & 1974 \\
\hline
\end{tabular}

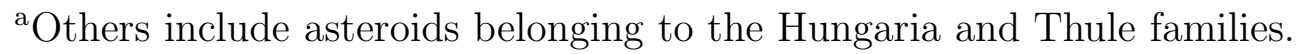


Table 2. Summary of the numbers $\left(N_{\mathrm{ID}}\right)$ and mean albedos $\left(\overline{p_{\mathrm{v}}}\right)$ in the five taxonomic types of total MBAs and MBAs larger than $20 \mathrm{~km}$ detected by $A K A R I$. Same as table 8 in Usui et al. (2011), but showing data for MBAs only. The number of asteroids with identified taxonomies is greater than that presented in Usui et al. (2011), thanks to Carvano et al. (2010).

\begin{tabular}{crcrl}
\hline \hline type & $N_{\mathrm{ID}}$ & $\overline{p_{\mathrm{v}}}$ & $N_{\mathrm{ID}}(d>20 \mathrm{~km})$ & $\overline{p_{\mathrm{v}}}(d>20 \mathrm{~km})$ \\
\hline $\mathrm{C}$ & 1150 & $0.071 \pm 0.040$ & 613 & $0.066 \pm 0.031$ \\
$\mathrm{~S}$ & 722 & $0.208 \pm 0.079$ & 341 & $0.192 \pm 0.060$ \\
$\mathrm{X}$ & 490 & $0.098 \pm 0.081$ & 368 & $0.094 \pm 0.073$ \\
$\mathrm{D}$ & 130 & $0.086 \pm 0.053$ & 87 & $0.077 \pm 0.041$ \\
$\mathrm{~V}$ & 4 & $0.297 \pm 0.131$ & 1 & 0.342 \\
\hline total & 2496 & 1410 & \\
\hline
\end{tabular}


Table 3. Mean albedo values $\left(\overline{p_{\mathrm{v}}}\right)$ of the subclasses of X-type asteroids, separated by main belt region: inner, middle, and outer. Numbers of AcuA asteroids in each category are given in parentheses.

\begin{tabular}{|c|c|c|c|}
\hline & $\mathrm{E}$ & M & $\mathrm{P}$ \\
\hline Inner & $\begin{array}{l}0.454 \pm 0.119 \\
(6)\end{array}$ & $\begin{array}{l}0.169 \pm 0.044 \\
(22)\end{array}$ & $\begin{array}{l}0.063 \pm 0.017 \\
(36)\end{array}$ \\
\hline Middle & $\begin{array}{l}0.397 \pm 0.076 \\
(6)\end{array}$ & $\begin{array}{l}0.166 \pm 0.041 \\
(56)\end{array}$ & $\begin{array}{l}0.057 \pm 0.018 \\
(106)\end{array}$ \\
\hline Outer & $\begin{array}{l}0.376 \pm 0.016 \\
(2)\end{array}$ & $\begin{array}{l}0.166 \pm 0.050 \\
(67)\end{array}$ & $\begin{array}{l}0.052 \pm 0.017 \\
(189)\end{array}$ \\
\hline \multicolumn{4}{|c|}{$d>20 \mathrm{~km}$} \\
\hline Inner & $\begin{array}{l}0.479 \\
(1)\end{array}$ & $\begin{array}{l}0.161 \pm 0.033 \\
(10)\end{array}$ & $\begin{array}{l}0.061 \pm 0.016 \\
(21)\end{array}$ \\
\hline Middle & $\begin{array}{l}0.387 \pm 0.081 \\
(5)\end{array}$ & $\begin{array}{l}0.165 \pm 0.039 \\
(42)\end{array}$ & $\begin{array}{l}0.054 \pm 0.017 \\
(77)\end{array}$ \\
\hline Outer & $\begin{array}{l}0.365 \\
(1)\end{array}$ & $\begin{array}{l}0.169 \pm 0.050 \\
(58)\end{array}$ & $\begin{array}{l}0.053 \pm 0.017 \\
(153)\end{array}$ \\
\hline
\end{tabular}


Table 4 . Large $(d>20 \mathrm{~km})$ E-type asteroids.

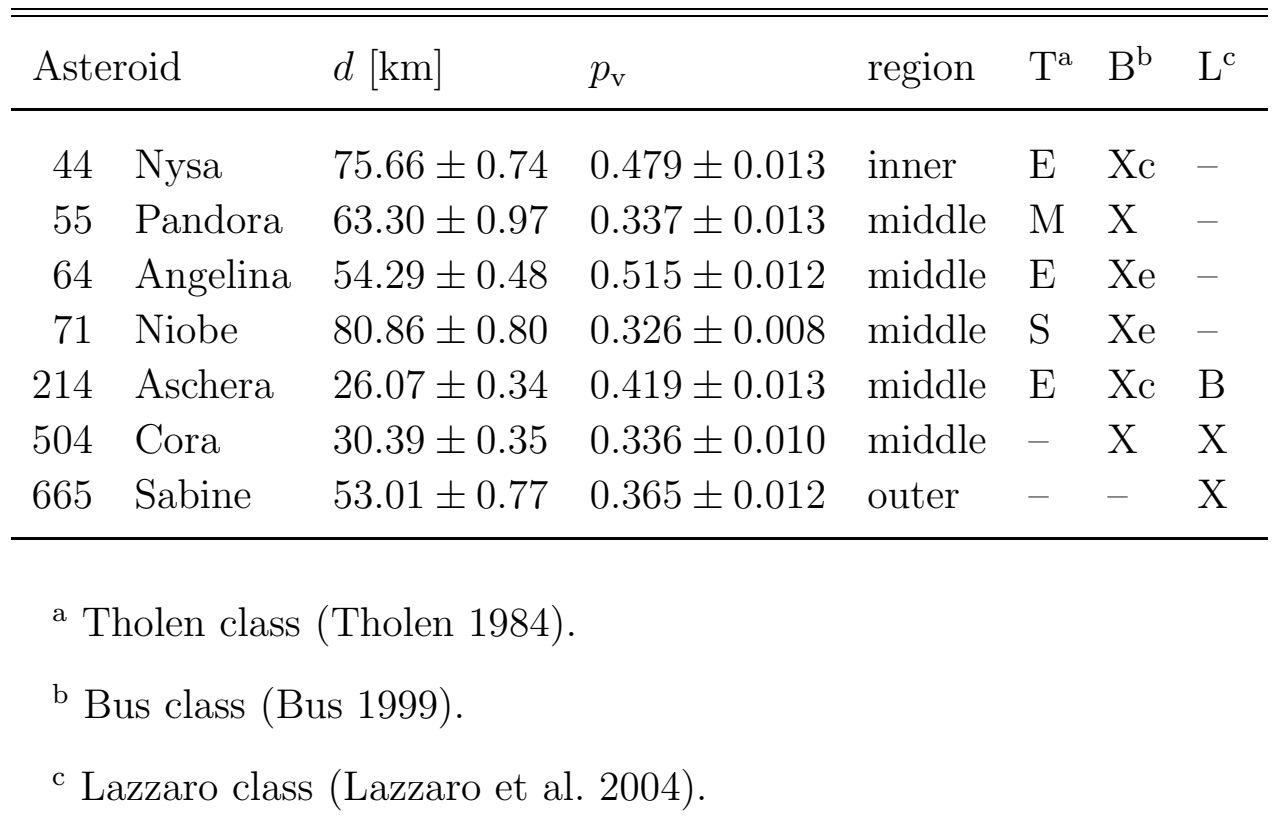


(a)

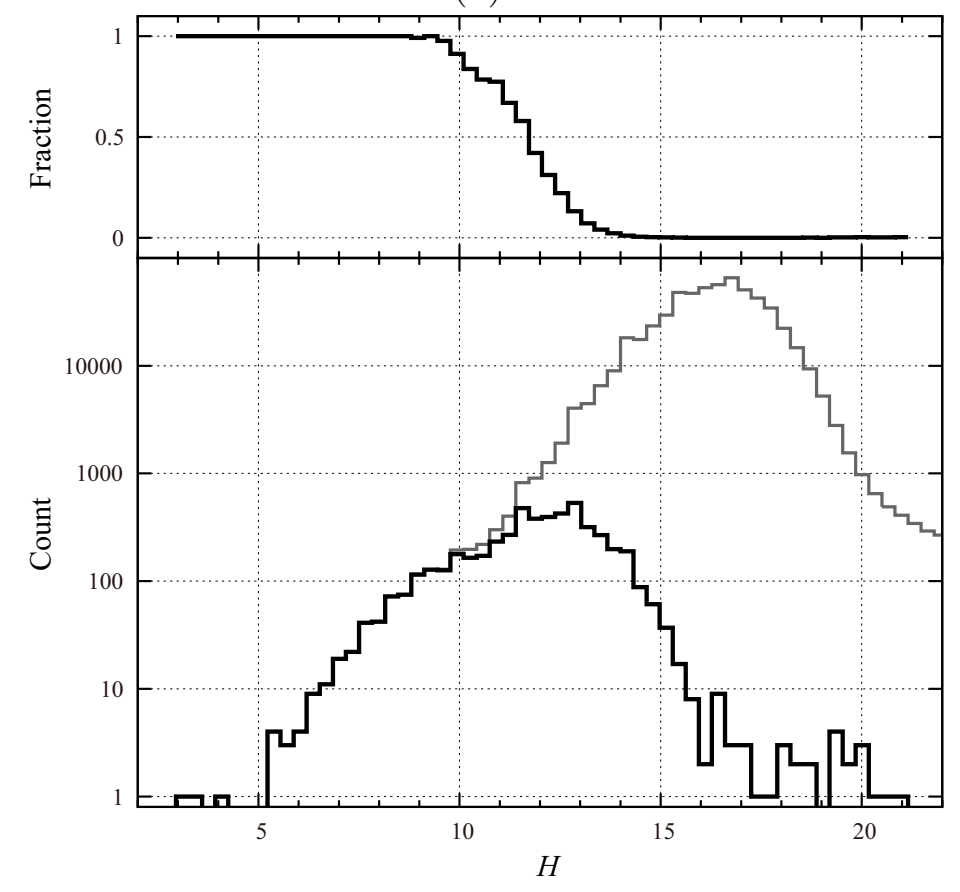

(b)

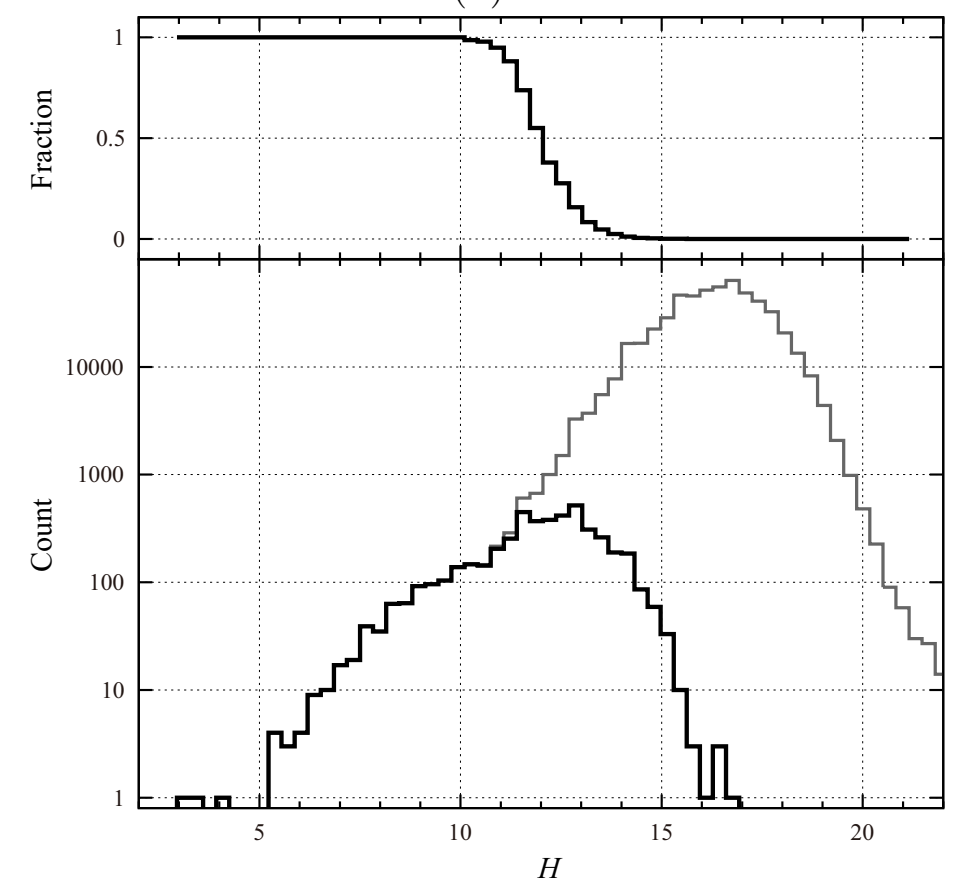

Fig. 1.- Distribution of absolute magnitude $(H)$ for asteroids detected by $A K A R I$ with known orbits. (a) total asteroids with semimajor axes smaller than 6 AU, (b) total MBAs. The upper panel of each figure shows the fraction of detected asteroids of the total known asteroids, and the lower panel shows the distribution of detected asteroids (black line) and that of total asteroids with known orbits(gray line). The bin size is set to 60 segments for $H$ range of 2 to 22 . 


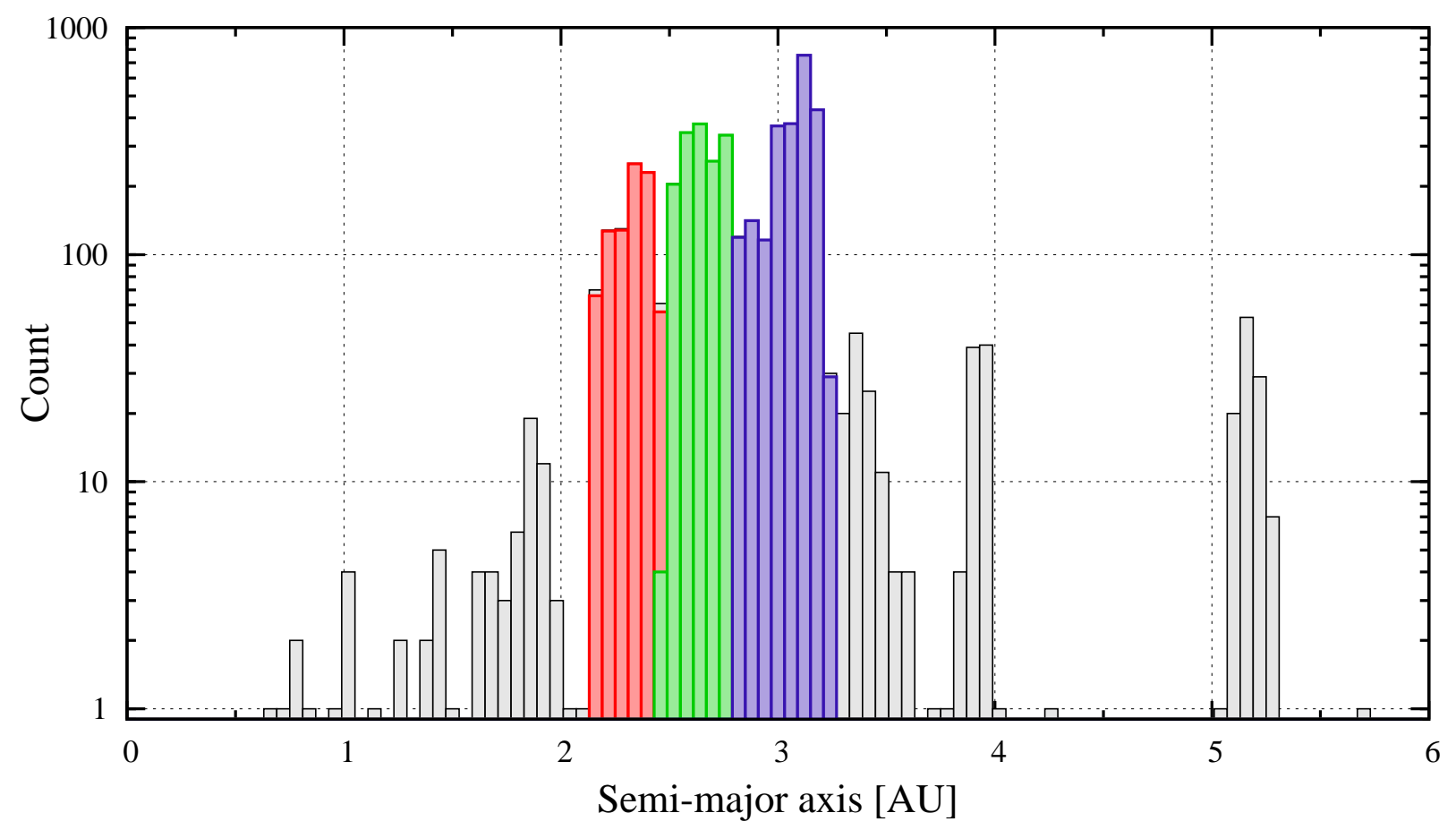

Fig. 2.- Histogram of the number of detected asteroids with $A K A R I$ as a function of the semimajor axis. Red, green, and blue boxes denote inner, middle, and outer region MBAs, respectively. The bin size is set to 100 segments for the semimajor axis range of 0 to 6 AU. 


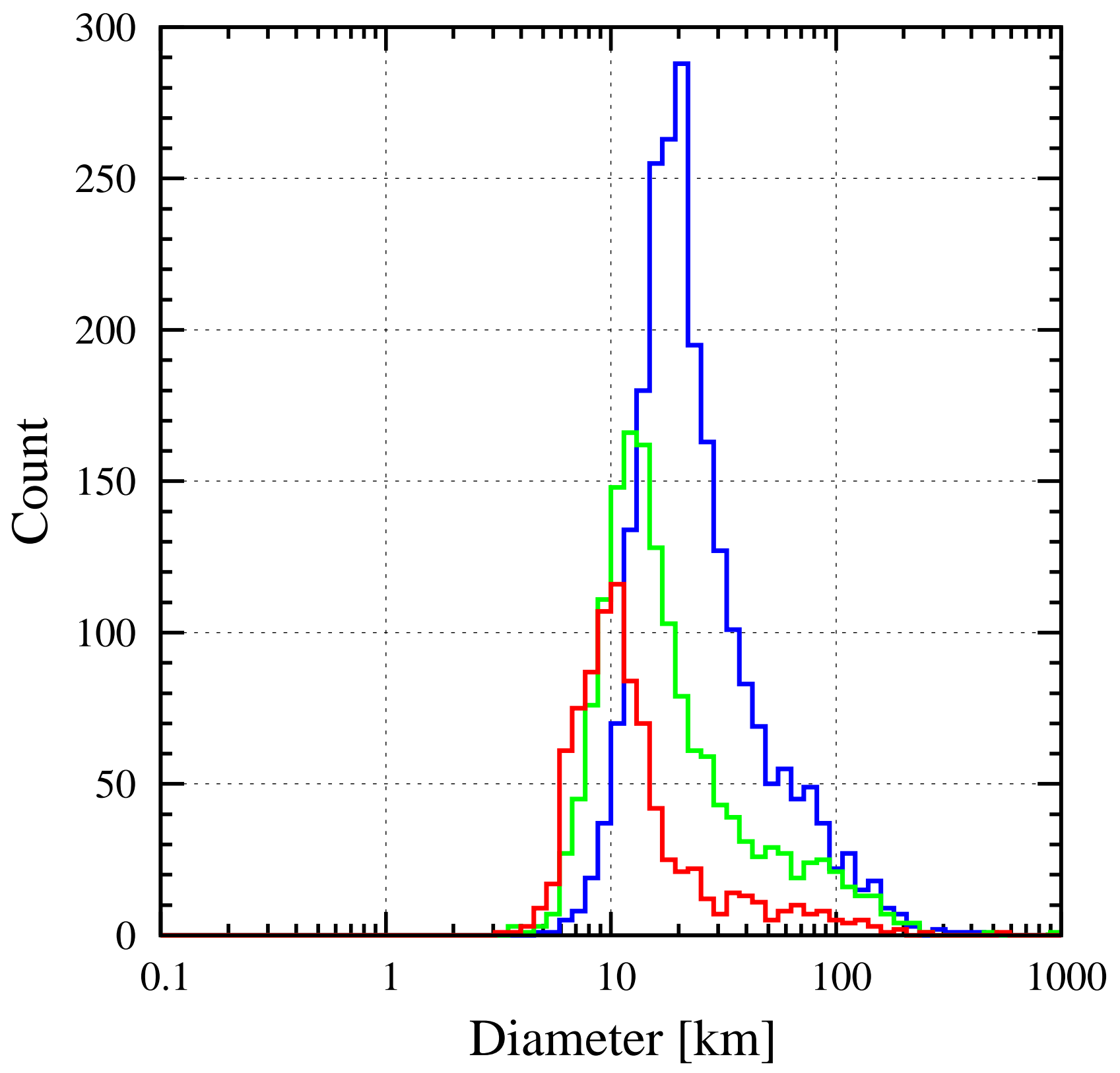

Fig. 3.- Histogram of the size distribution of AcuA MBAs. Red, green, and blue lines denote inner, middle, and outer region MBAs, respectively. 
(a) MBAs total

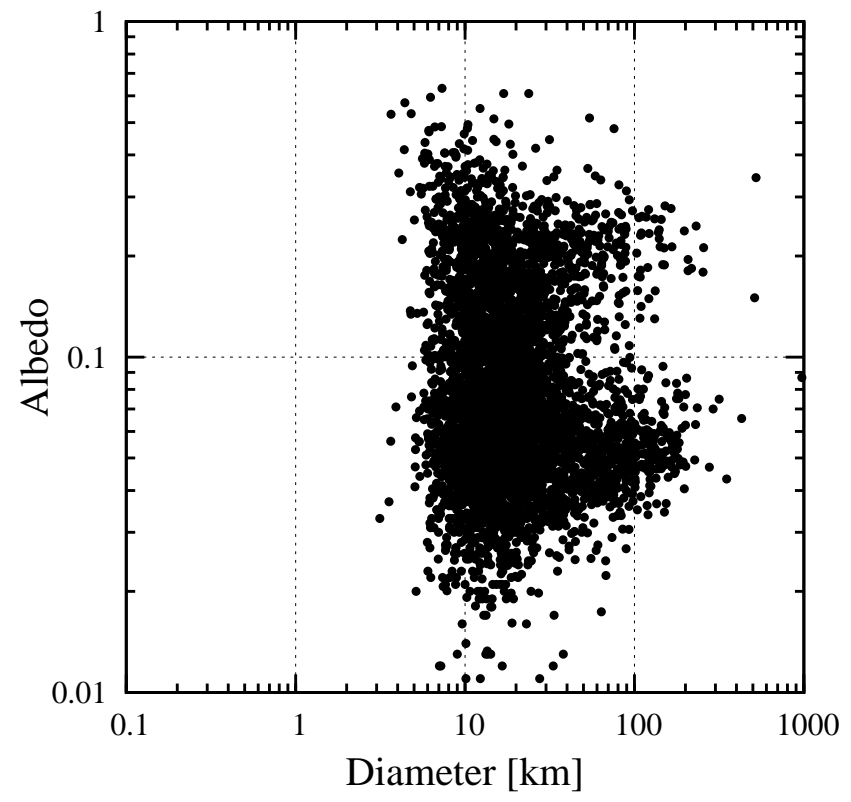

(c) middle MBAs

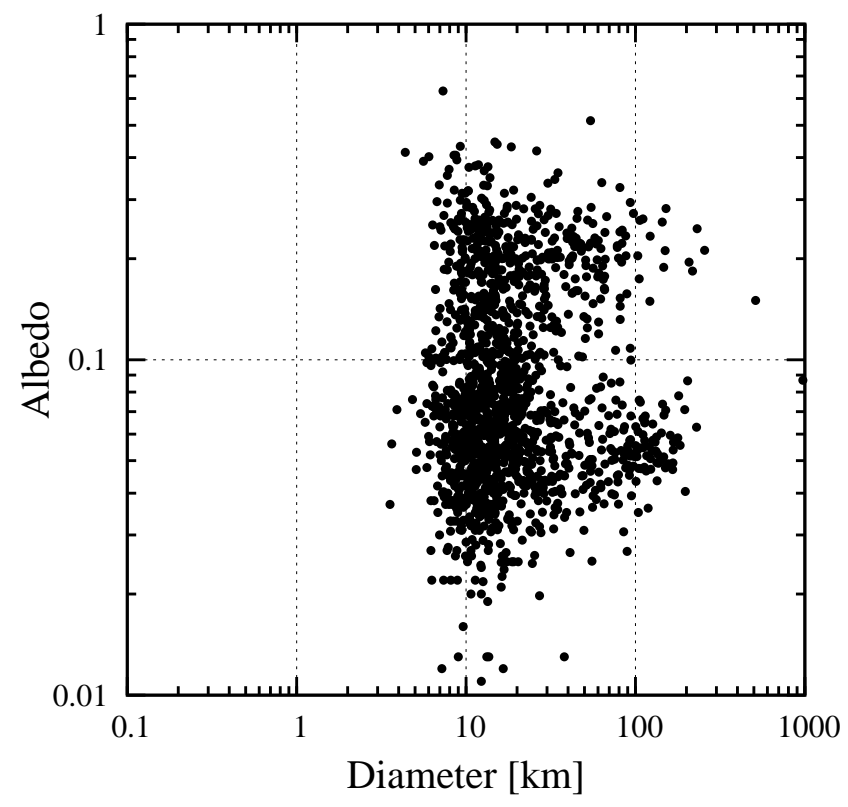

(b) inner MBAs

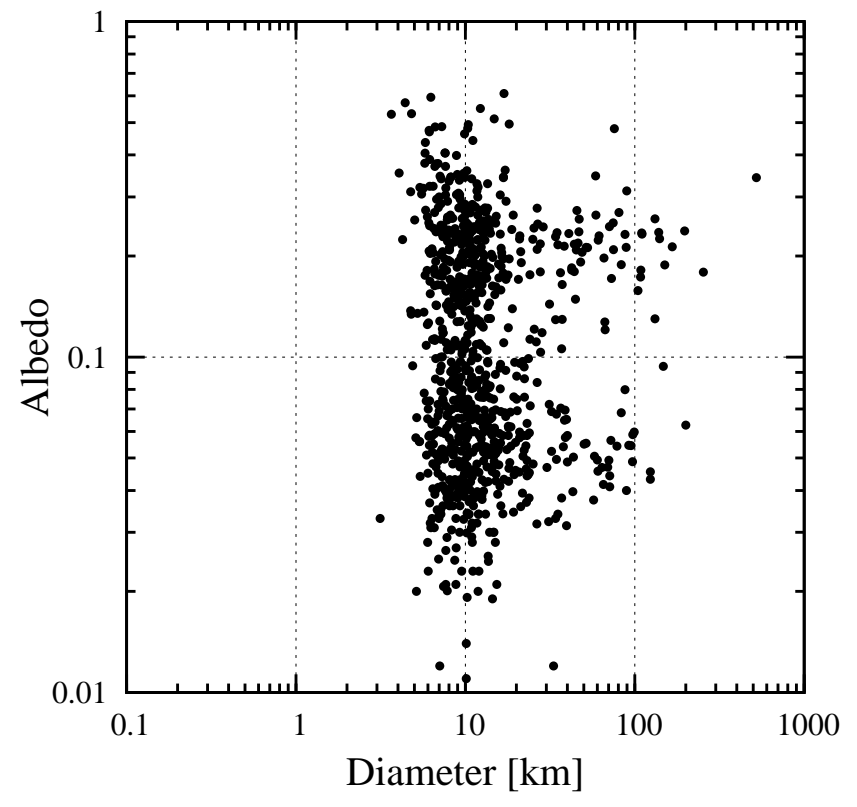

(d) outer MBAs

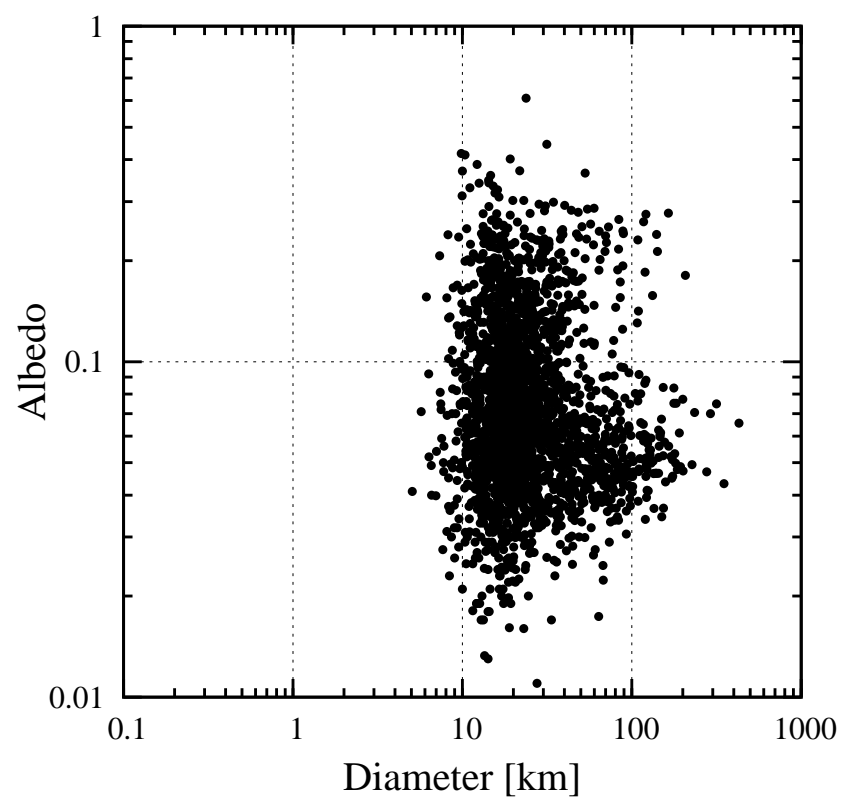

Fig. 4.- Size-albedo distributions for AcuA MBAs: (a) total population, and (b) inner, (c) middle, and (d) outer region MBAs. 
(a) C-type

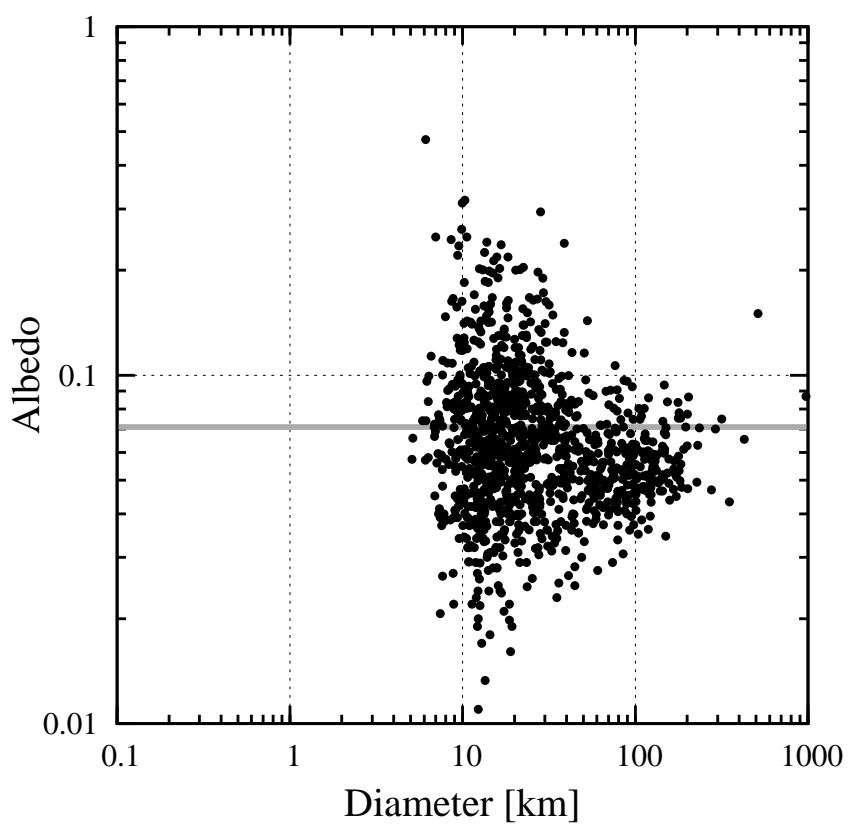

(c) X-type

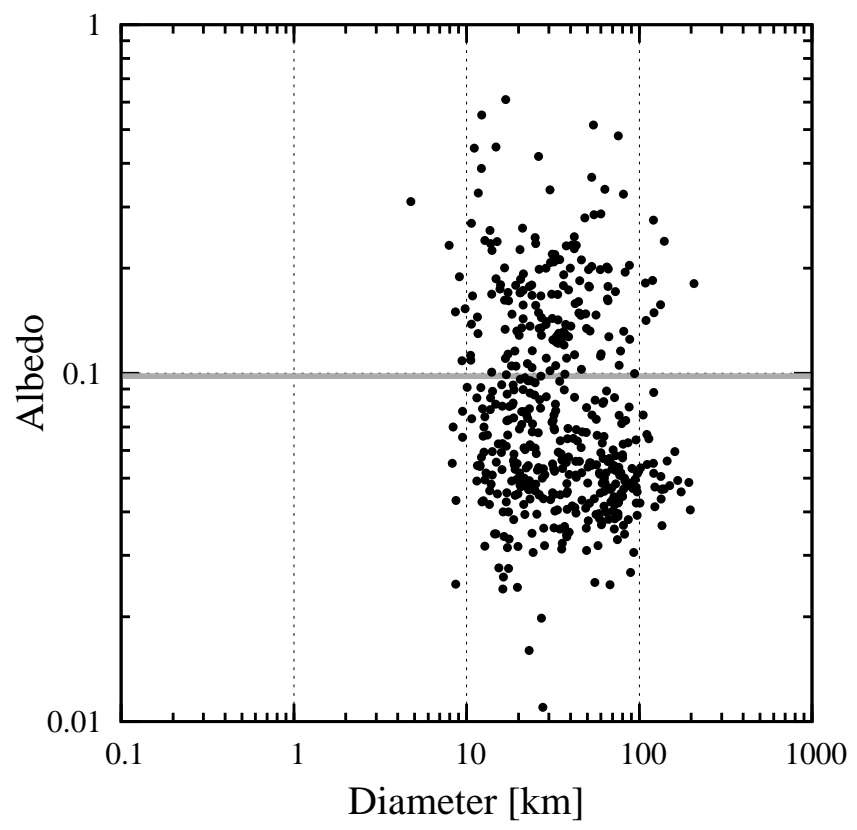

(b) S-type

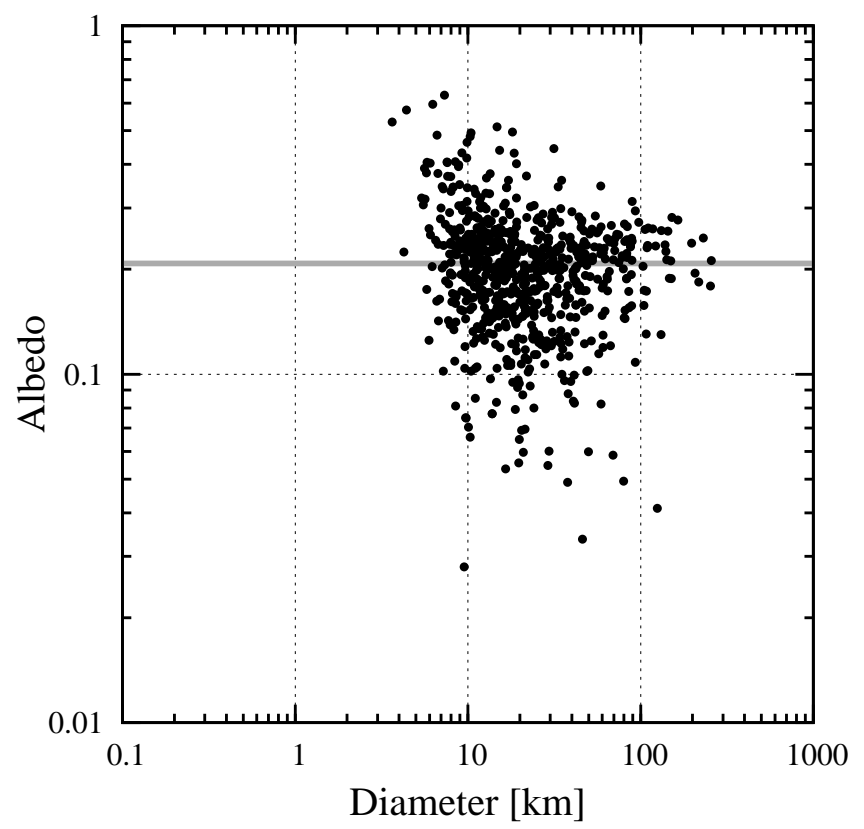

(d) D-type

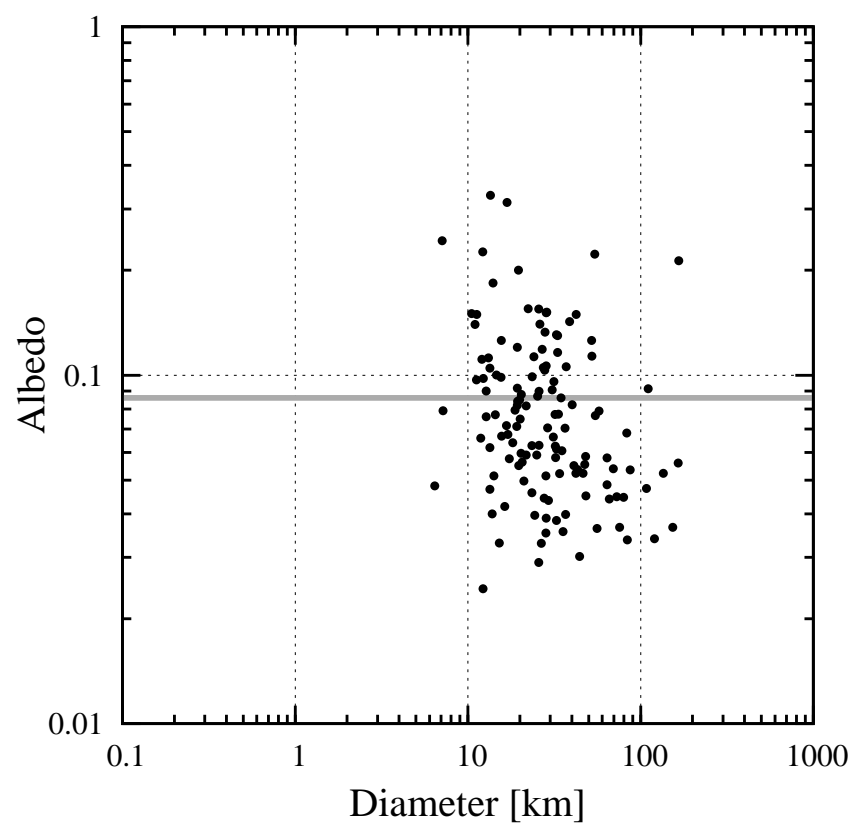

Fig. 5.- Size-albedo distributions for each taxonomic type of AcuA MBAs. Solid gray lines indicate the mean albedo values (see Table 2). 


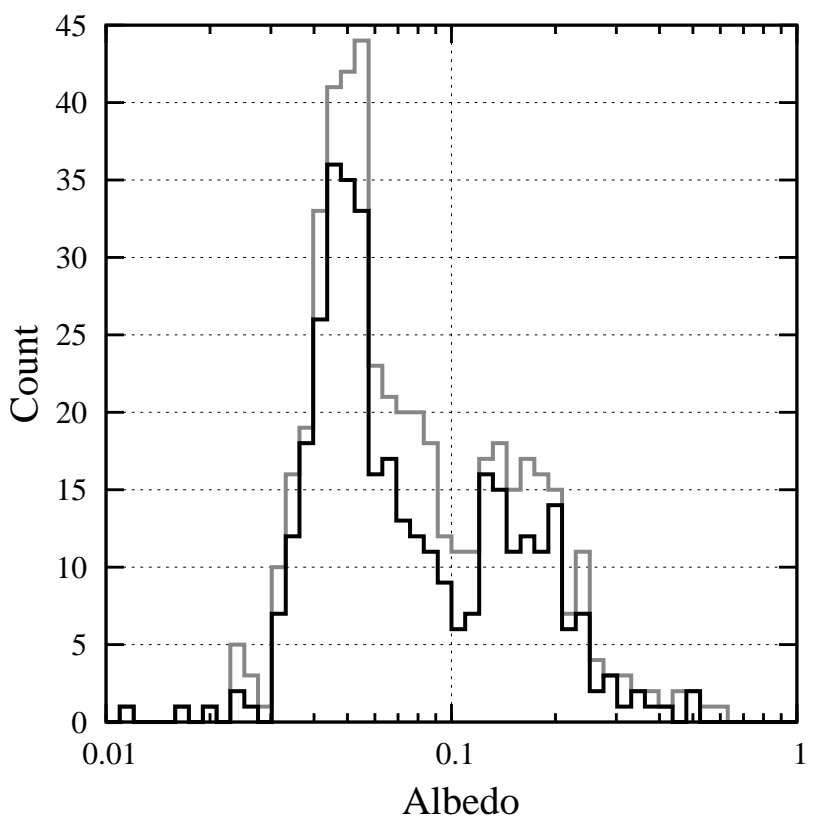

Fig. 6. - Histogram of albedo values for AcuA X-type MBAs. Gray and black lines denote all X-types, and X-types larger than $20 \mathrm{~km}$, respectively. The bin size is set at 50 segments for the albedo range of $0.01-1.0$ in the logarithmic scale. 
(a) $0.1 \leq d<20 \mathrm{~km}$

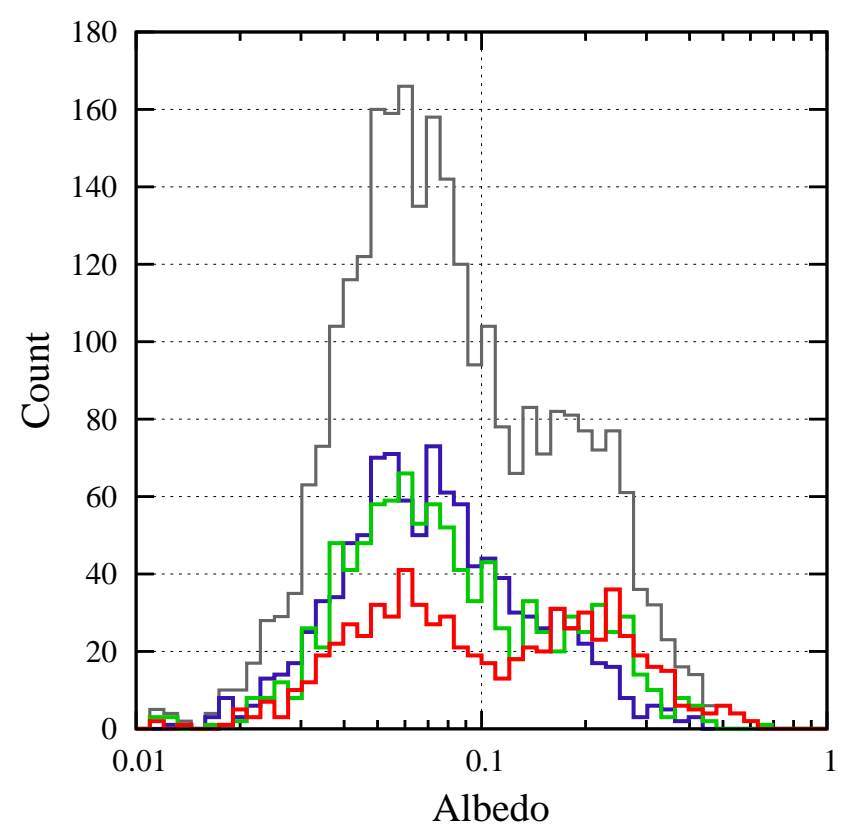

(c) $d \geq 100 \mathrm{~km}$

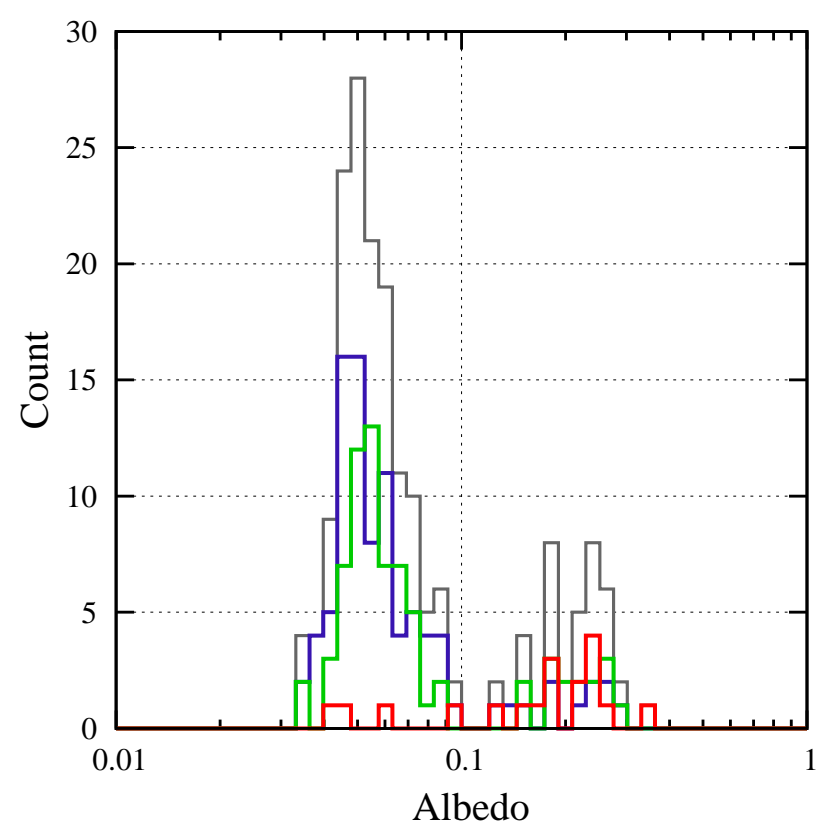

(b) $20 \leq d<100 \mathrm{~km}$

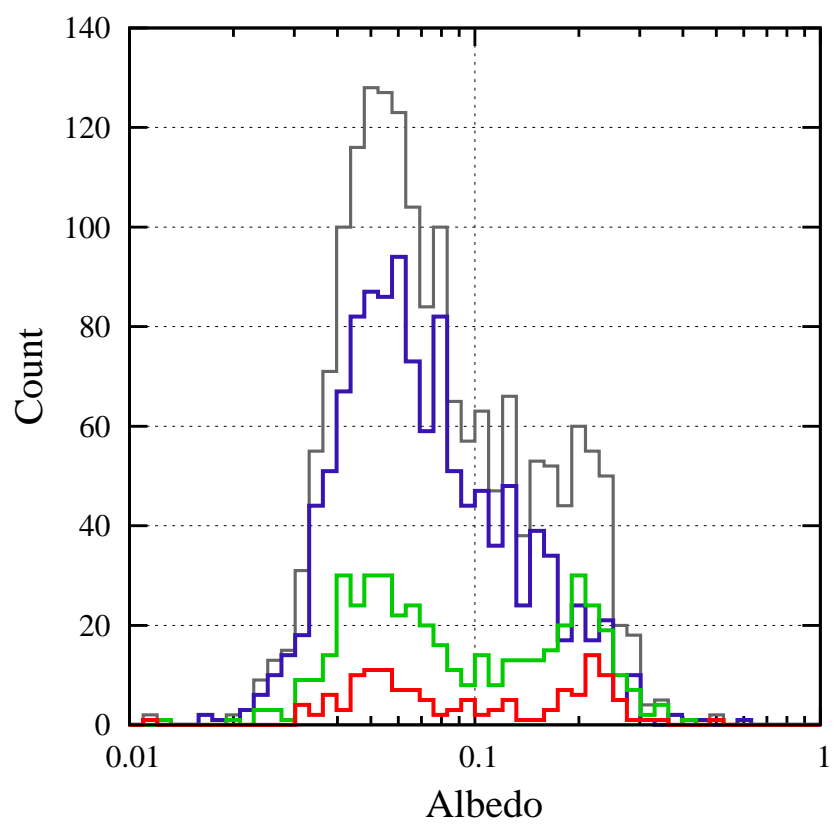

Fig. 7.- Histogram of albedo values for MBAs detected by $A K A R I$ for three size classes (diameters, $d$, in km): (a) $0.1 \leq d<20$; (b) $20 \leq d<100$; (c) $d \geq 100$. Red, green, blue, and gray lines denote inner region, middle region, outer region, and total MBAs, respectively. The bin size is set at 50 segments for the albedo range of 0.01-1.0 in the logarithmic scale. 
(a)

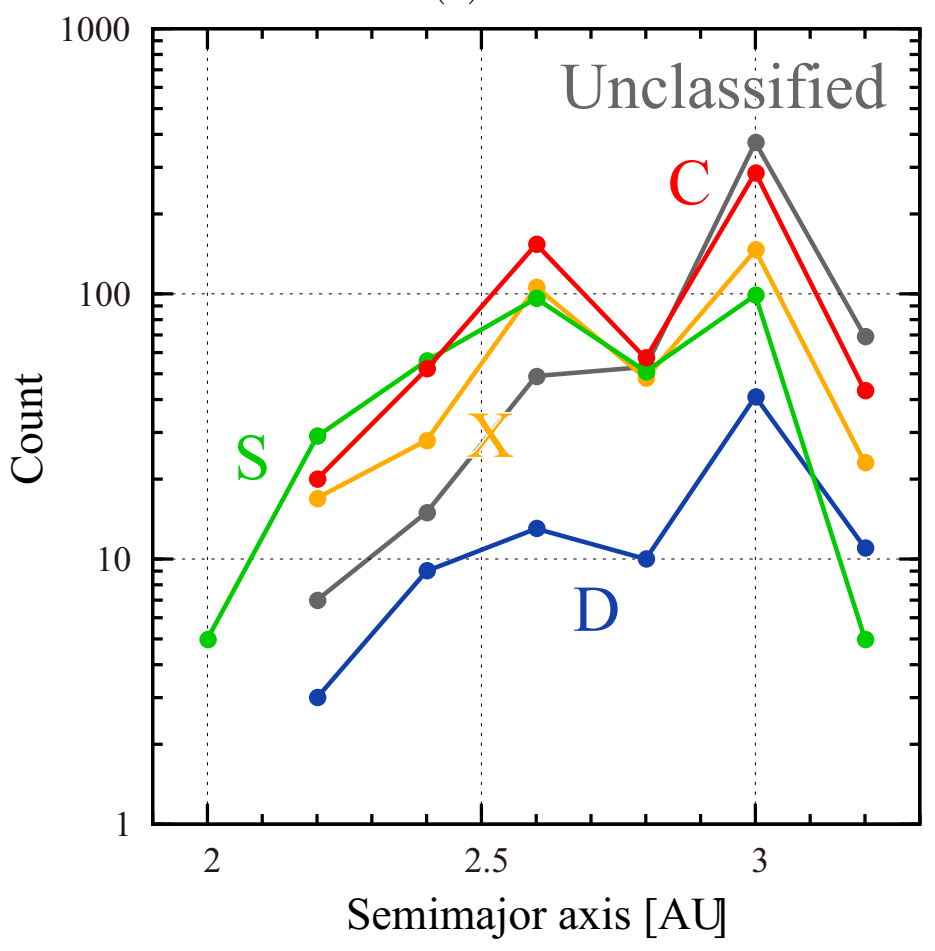

(b)

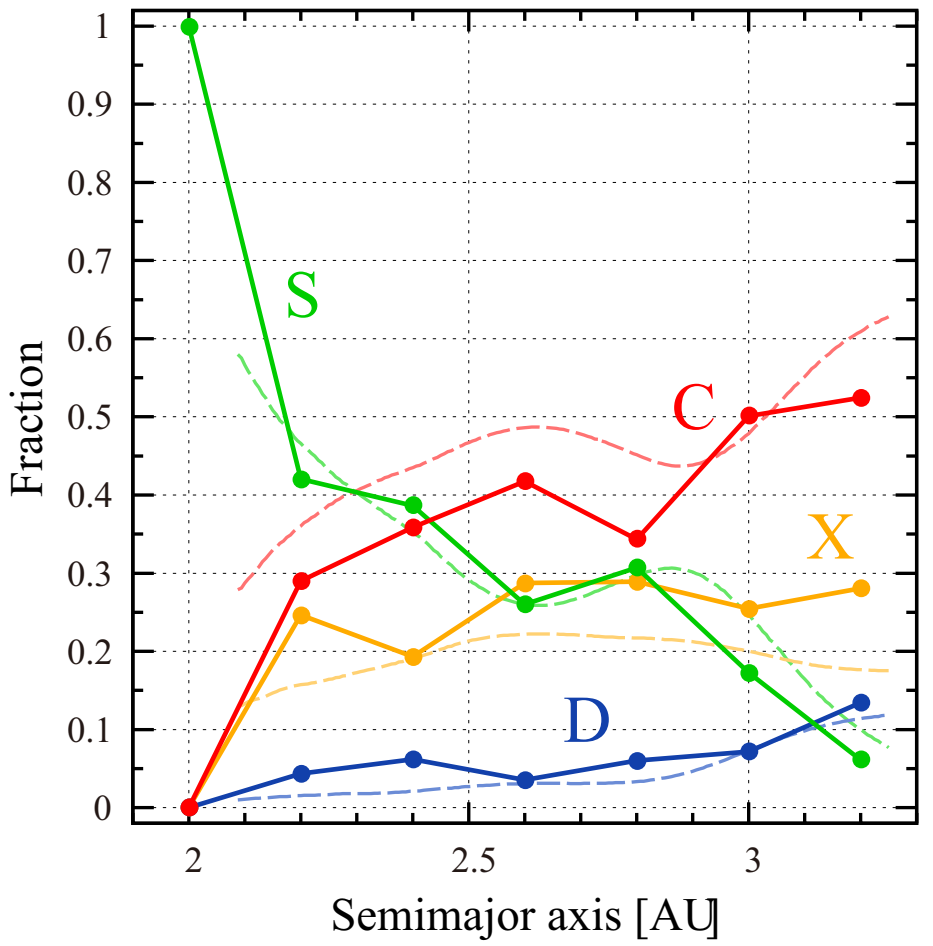

Fig. 8. - Histograms showing the numerical distribution (a) and fractional distribution (b) of MBAs with $d \geq 20 \mathrm{~km}$ for each of the taxonomic types. Bold lines in (b) show AcuA data (this study) and dashed lines show data from Bus \& Binzel (2002). Red, green, yellow, and blue denote $\mathrm{C}-, \mathrm{S}-, \mathrm{X}-$, and D-type asteroids, respectively. 
(a)

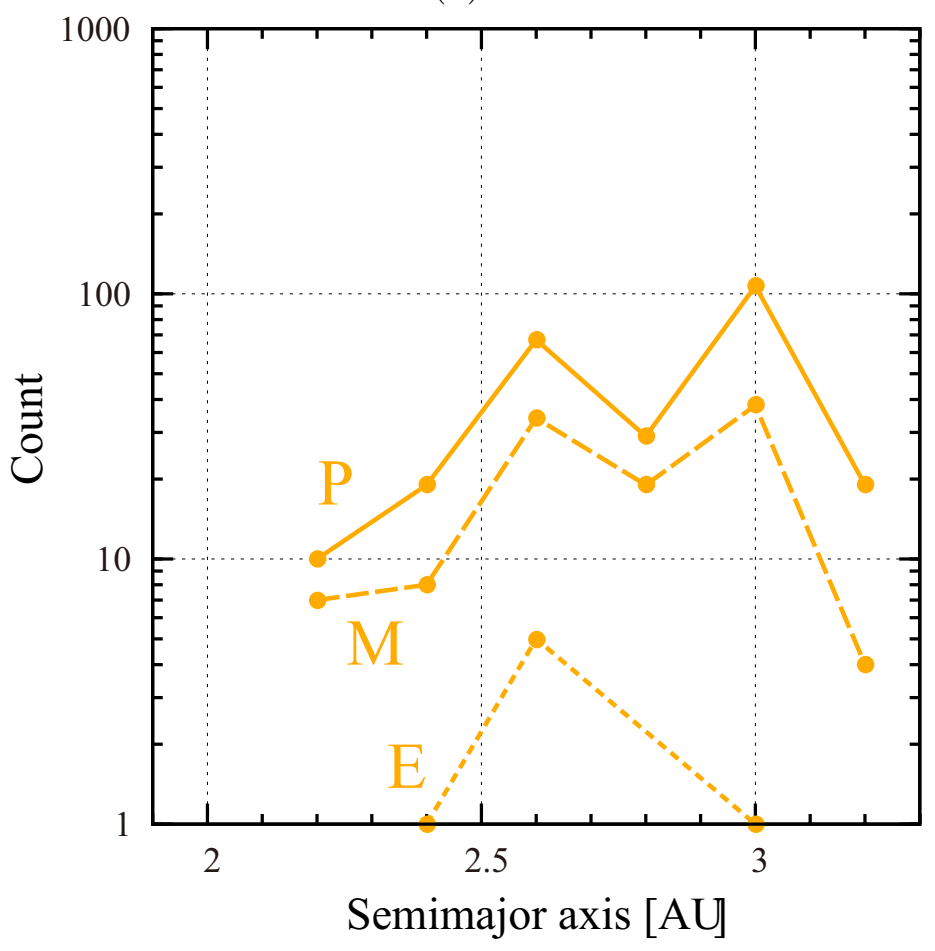

(b)

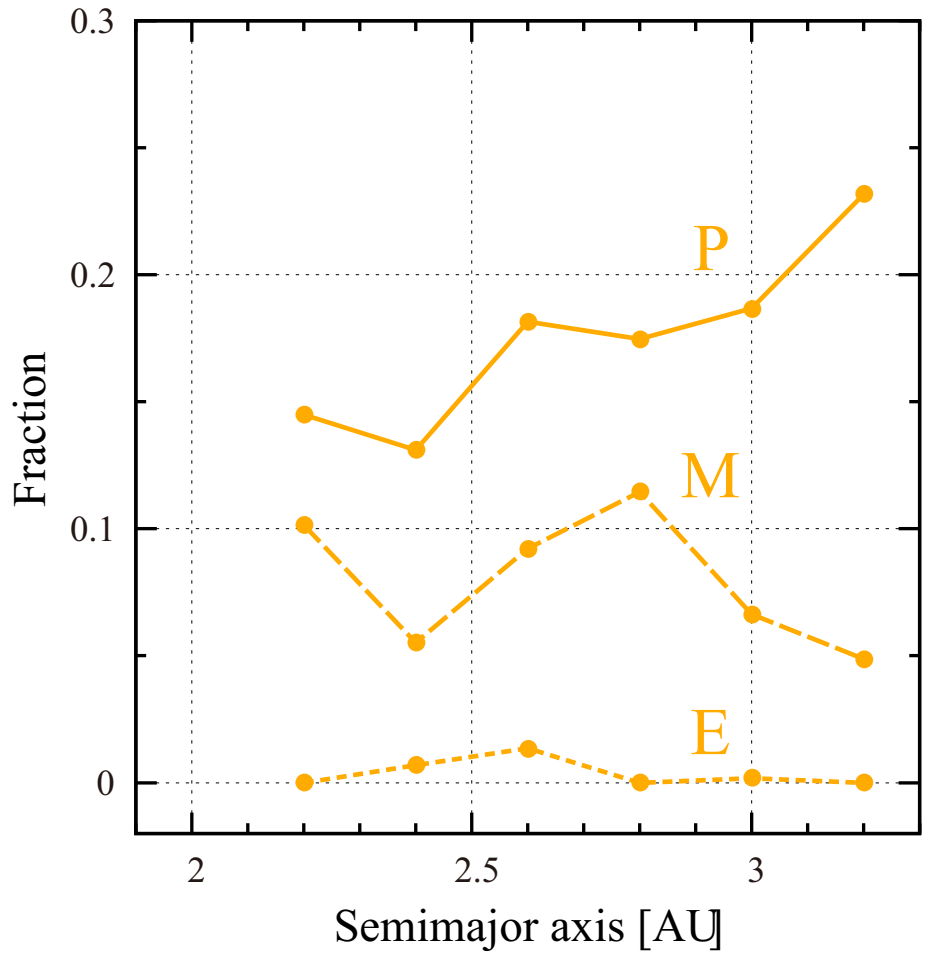

Fig. 9.- Histograms showing the numerical distribution (a) and fractional distribution (b) of X-type MBAs with $d \geq 20 \mathrm{~km}$. Dotted, dashed, and bold lines denote E-, M-, and P-type asteroids, respectively. 
(a) total

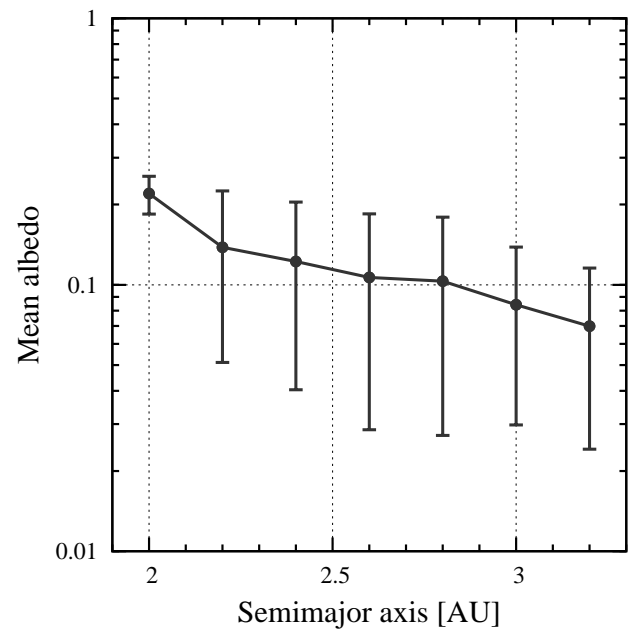

(b) C-type

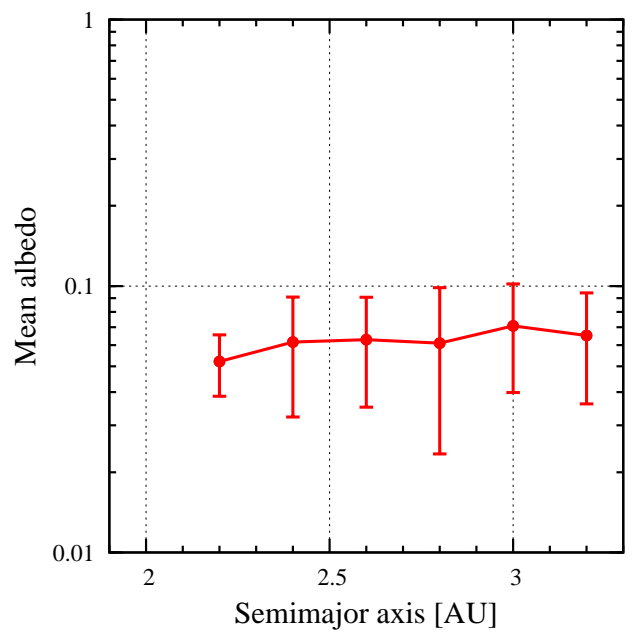

(d) X-type

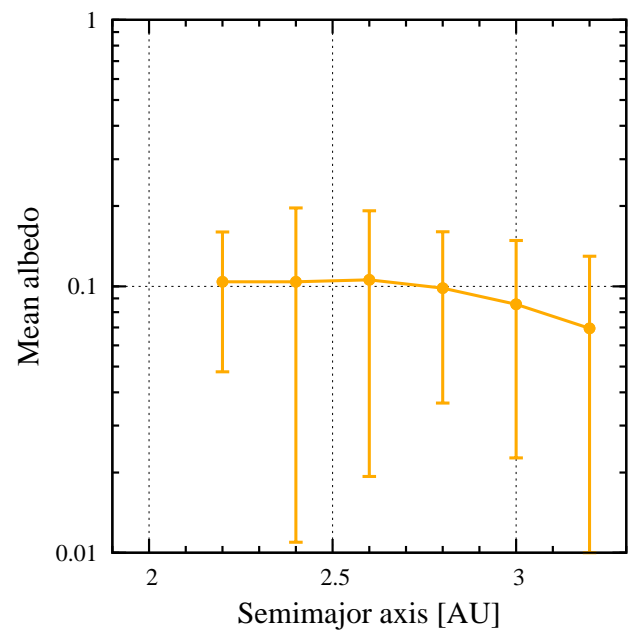

(c) S-type

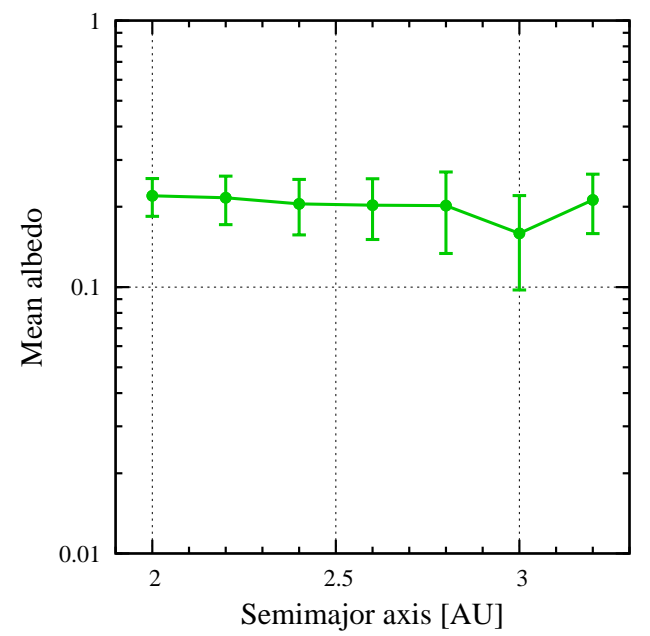

(e) D-type

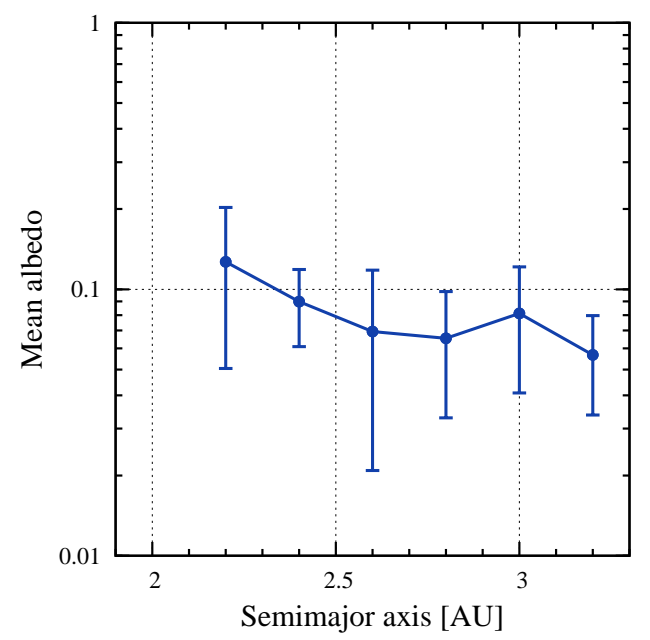

Fig. 10.- Mean albedo as a function of the semimajor axis for MBAs with $d \geq 20 \mathrm{~km}$ for 


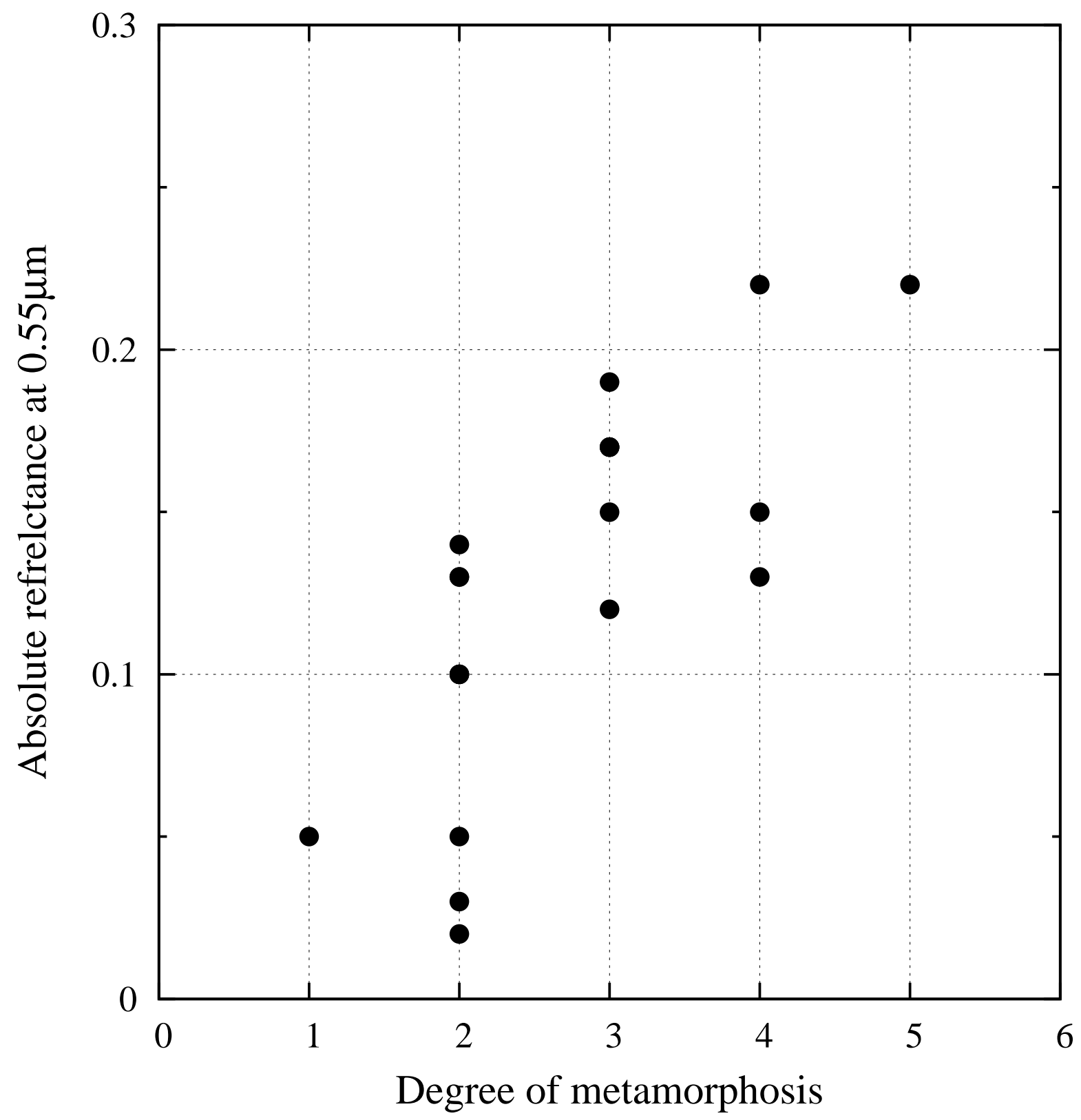

Fig. 11. - Absolute reflectance at $0.55 \mu \mathrm{m}$ as a function of the degree of metamorphosis/alteration of meteorites measured in laboratories; compiled by Clark et al. (2009). 
Table 5. Summary of the numbers, mean albedos, and albedo variations of 784 asteroids detected by $A K A R I$, classified according to Tholen taxonomy. The classification is based on the references in Usui et al. (2011), appendix 4. Note: Asteroids belonging to Q (S-type) and $\mathrm{W}$ (X-type) classes are not detected by $A K A R I$.

\begin{tabular}{|c|c|c|c|c|}
\hline type $^{\mathrm{a}}$ & $\operatorname{class}^{b}$ & $N_{\mathrm{ID}}{ }^{\mathrm{c}}$ & $\overline{p_{\mathrm{v}}} \quad \mathrm{d}$ & $\sigma\left(\overline{p_{\mathrm{v}}}\right)^{\mathrm{e}}$ \\
\hline \multirow[t]{4}{*}{$\mathrm{C}$} & B & 13 & 0.113 & 0.069 \\
\hline & $\mathrm{C}$ & 178 & 0.061 & 0.028 \\
\hline & $\mathrm{F}$ & 35 & 0.058 & 0.023 \\
\hline & $\mathrm{G}$ & 10 & 0.073 & 0.018 \\
\hline \multirow[t]{5}{*}{$\mathrm{S}$} & $\mathrm{A}$ & 5 & 0.282 & 0.101 \\
\hline & $\mathrm{R}$ & 1 & 0.277 & - \\
\hline & $\mathrm{S}$ & 339 & 0.213 & 0.071 \\
\hline & $\mathrm{O}$ & 1 & 0.256 & - \\
\hline & $\mathrm{K}$ & 23 & 0.143 & 0.039 \\
\hline \multirow[t]{3}{*}{ X } & $E$ & 7 & 0.559 & 0.140 \\
\hline & M & 38 & 0.175 & 0.052 \\
\hline & $\mathrm{P}$ & 39 & 0.049 & 0.018 \\
\hline \multirow[t]{2}{*}{$\mathrm{D}$} & $\mathrm{D}$ & 84 & 0.061 & 0.030 \\
\hline & $\mathrm{T}$ & 7 & 0.075 & 0.023 \\
\hline \multirow[t]{2}{*}{$\mathrm{V}$} & $\mathrm{V}$ & 3 & 0.293 & 0.150 \\
\hline & $\mathrm{J}$ & 1 & 0.436 & - \\
\hline
\end{tabular}

aTaxonomic type of asteroids.

bTaxonomic class of asteroids determined by Tholen (1984); Lazzaro et al. (2004).

${ }^{\mathrm{c}}$ Number of detections by $A K A R I$.

dMean geometric albedo in optical.

eAlbedo variations. 
Table 6. Summary of the numbers, mean albedos, and albedo variations of 894 asteroids detected by $A K A R I$, classified according to Bus taxonomy. Classification is based on the references in Usui et al. (2011), appendix 4.

\begin{tabular}{|c|c|c|c|c|}
\hline type $\mathrm{a}^{\mathrm{a}}$ & class $^{b}$ & $N_{\mathrm{ID}}{ }^{\mathrm{c}}$ & $\overline{p_{\mathrm{v}}} \quad \mathrm{d}$ & $\sigma\left(\overline{p_{\mathrm{v}}}\right)^{\mathrm{e}}$ \\
\hline \multirow[t]{6}{*}{ C } & B & 46 & 0.092 & 0.056 \\
\hline & $\mathrm{C}$ & 121 & 0.072 & 0.043 \\
\hline & $\mathrm{Cb}$ & 25 & 0.075 & 0.058 \\
\hline & $\mathrm{Cg}$ & 8 & 0.069 & 0.029 \\
\hline & $\mathrm{Cgh}$ & 11 & 0.097 & 0.041 \\
\hline & $\mathrm{Ch}$ & 128 & 0.063 & 0.023 \\
\hline \multirow[t]{13}{*}{$\bar{S}$} & $\overline{\mathrm{A}}$ & 9 & 0.298 & 0.143 \\
\hline & K & 28 & 0.154 & 0.065 \\
\hline & $\mathrm{L}$ & 22 & 0.139 & 0.038 \\
\hline & $\mathrm{Ld}$ & 7 & 0.137 & 0.060 \\
\hline & $\mathrm{O}$ & 2 & 0.334 & 0.110 \\
\hline & $\mathrm{Q}$ & 2 & 0.353 & 0.001 \\
\hline & $\mathrm{R}$ & 1 & 0.277 & - \\
\hline & S & 185 & 0.233 & 0.069 \\
\hline & $\mathrm{Sa}$ & 14 & 0.224 & 0.076 \\
\hline & Sk & 14 & 0.239 & 0.059 \\
\hline & $\mathrm{Sl}$ & 28 & 0.218 & 0.051 \\
\hline & $\mathrm{Sq}$ & 16 & 0.327 & 0.201 \\
\hline & $\mathrm{Sr}$ & 1 & 0.360 & - \\
\hline \multirow[t]{4}{*}{$\mathrm{X}$} & $\bar{X}$ & 101 & 0.119 & 0.079 \\
\hline & $\mathrm{Xc}$ & 49 & 0.100 & 0.092 \\
\hline & $\mathrm{Xe}$ & 21 & 0.286 & 0.201 \\
\hline & $\mathrm{Xk}$ & 35 & 0.111 & 0.132 \\
\hline \multirow[t]{2}{*}{$\bar{D}$} & $\mathrm{D}$ & 7 & 0.086 & 0.056 \\
\hline & $\mathrm{T}$ & 11 & 0.054 & 0.009 \\
\hline $\mathrm{V}$ & $\overline{\mathrm{V}}$ & 2 & 0.318 & 0.035 \\
\hline
\end{tabular}


aTaxonomic type of asteroids.

bTaxonomic class of asteroids determined by Bus (1999); Lazzaro et al. (2004).

${ }^{\mathrm{c}}$ Number of detections by $A K A R I$.

${ }^{\mathrm{d}}$ Mean geometric albedo in optical.

eAlbedo variations. 
Table 7. Summary of the numbers, mean albedos, and albedo variations of 1347 asteroids detected by $A K A R I$, classified according to the Carvano taxonomy. Note: Asteroids belonging to CD, CO, CS, AV, LA, LQ, OV, QV, SO, SV, XS, DS classes are not detected by $A K A R I$.

\begin{tabular}{|c|c|c|c|c|}
\hline type $e^{a}$ & class $^{b}$ & $N_{\text {ID }}{ }^{\mathrm{c}}$ & $\overline{p_{\mathrm{v}}} \quad \mathrm{d}$ & $\sigma\left(\overline{p_{\mathrm{v}}}\right)^{\mathrm{e}}$ \\
\hline \multirow[t]{4}{*}{$\mathrm{C}$} & $\mathrm{C}$ & 701 & 0.069 & 0.040 \\
\hline & CL & 1 & 0.149 & - \\
\hline & CQ & 1 & 0.597 & - \\
\hline & CX & 66 & 0.061 & 0.034 \\
\hline \multirow[t]{10}{*}{$\bar{S}$} & $\bar{A}$ & 2 & 0.268 & 0.045 \\
\hline & $\mathrm{AQ}$ & 1 & 0.194 & - \\
\hline & $\mathrm{L}$ & 80 & 0.173 & 0.091 \\
\hline & LS & 32 & 0.194 & 0.059 \\
\hline & $\mathrm{O}$ & 3 & 0.135 & 0.115 \\
\hline & Q & 3 & 0.269 & 0.127 \\
\hline & $\mathrm{QO}$ & 1 & 0.054 & - \\
\hline & $\mathrm{S}$ & 120 & 0.218 & 0.072 \\
\hline & $\mathrm{SA}$ & 1 & 0.232 & - \\
\hline & SQ & 2 & 0.217 & 0.027 \\
\hline \multirow[t]{3}{*}{$\mathrm{X}$} & X & 199 & 0.084 & 0.080 \\
\hline & XD & 10 & 0.080 & 0.050 \\
\hline & XL & 9 & 0.123 & 0.066 \\
\hline \multirow[t]{2}{*}{$\mathrm{D}$} & $\mathrm{D}$ & 111 & 0.064 & 0.026 \\
\hline & $\mathrm{DL}$ & 3 & 0.171 & 0.065 \\
\hline $\mathrm{V}$ & V & 1 & 0.2840 & - \\
\hline
\end{tabular}

${ }^{a}$ Taxonomic type of asteroids.

b Taxonomic class of asteroids determined by Carvano et al. (2010).

${ }^{\mathrm{c}}$ Number of detections by $A K A R I$. 
${ }^{\mathrm{d}}$ Mean geometric albedo in optical.

${ }^{\mathrm{e}}$ Albedo variations. 


\section{REFERENCES}

Allen, D. A. 1970, Nature, 227, 158

Allen, D. A. 1971, in Physical Studies of Minor Planets, ed. T. Gehrels (Washington: National Aeronautics and Space Administration SP-267), 41

Bell, J. F., et al. 1989, in Asteroids II, ed. R. P. Binzel et al. (Tucson: University of Arizona Press), 921

Binzel, R. P., et al. 2010, Nature, 463, 331

Binzel, R. P., Rivkin, A. S., Stuart, J. S., Harris, A. W., Bus, S. J., \& Burbine, T. H. 2004, Icarus, 170,259

Bottke, W. F., Jr., Durda, D. D., Nesvorný, D., Jedicke, R., Morbidelli, A., Vokrouhlický, D., \& Levison, H. F. 2005, Icarus, 179, 63

Bottke, W. F., Jr., Cellino, A., Paolicchi, P., \& Binzel, R. P. 2002, in Asteroids III, ed. W. F. Bottke et al. (Tucson: University of Arizona Press), 3

Bottke, W. F., Jr., Vokrouhlickyý, D., Rubincam, D. P., \& Broz, M. 2002, in Asteroids III, ed. W. F. Bottke et al. (Tucson: University of Arizona Press), 395

Bowell, E., Muinonen, K., \& Wasserman, L. H. 1994, in Asteroids, Comets, Meteors 1993, ed. A. Milani et al. (Dordrecht: Kluwer Academic Publishers), 477

Brownlee, D. E., et al. 2003, Journal of Geophysical Research, 108, 8111

Burbine, T. H., Rivkin, A. S., Noble, S. K., Mothé-Diniz, T., Bottke, W. F., McCoy, T. J., Dyar, M. D., \& Thomas, C.A. 2008, in Reviews in Mineralogy \& Geochemistry 68, 273

Bus, S. J. 1999, PhD thesis, Massachusetts Institute of Technology

Bus, S. J., \& Binzel, R. P. 2002, Icarus, 158, 146

Carvano, J. M., Hasselmann, P. H., Lazzaro, D., \& Mothé-Diniz, T. 2010, A\&A, 510, A43

Carvano, J. M., Mothé-Diniz, T., \& Lazzaro, D. 2003, Icarus, 161, 356

Chapman, C. R., Morrison, D., \& Zellner, B. 1975, Icarus, 25, 104

Cheng, A. F., et al. 1997, Journal of Geophysical Research, 102, 23695 
Clark, B. E., et al. 2004, AJ, 128, 3070

Clark, B. E., et al. 2009, Icarus, 202, 119

Davis, D. R., Chapman, C. R., Greenberg, R., Weidenschilling, S. J., \& Harris, A. W. 1979, in Asteroids (Tucson: University of Arizona Press), 528

Davis, D. R., Durda, D. D., Marzari, F., Campo Bagatin, A., \& Gil-Hutton, R. 2002, in Asteroids III, ed. W. F. Bottke et al. (Tucson: University of Arizona Press), 545

Drummond, J. D., Cocke, W. J., Hege, E. K., \& Strittmatter, P. A. 1985, Icarus, 61, 132

Drummond, J., Christou, J., \& Nelson, J. 2009, Icarus, 202, 147

Durech, J., et al. 2011, Icarus, 214, 652

Fornasier, S., Clark, B. E., \& Dotto, E. 2011, Icarus, 214, 131

Fowler, J. W., \& Chillemi, J. R. 1992, Phillips Lab. Tech. Rep., 2049, 17

Froeschle, Cl., \& Greenberg, R. 1989, in Asteroids II, ed. R. P. Binzel et al. (Tucson: University of Arizona Press), 827

Fujiwara, A. 1982, Icarus, 52, 434

Fujiwara, A., et al. 2006, Science, 312, 1330

Gaffey, M. J., Cloutis, E. A., Kelley, M. S.,\& Reed, K. L. 2002, in Asteroids III, ed. W. F. Bottke et al. (Tucson: University of Arizona Press), 138

Glassmeier, K.-H., Boehnhardt, H., Koschny, D., Kührt, E., \& Richter, I. 2007, Space Science Reviews, 128, 1

Gradie, J. C., Chapman, C. R., \& Williams, J. G. 1979, in Asteroids (Tucson: University of Arizona Press), 359

Greenwood, R. C., Franchi, I. A., Kearsley, A. T., \& Alard, O. 2010, Geochimica et Cosmochimica Acta, 74, 1684

Hirata, N., \& Ishiguro, M. 2011, 42nd Lunar and Planetary Science Conference, 1821

Hiroi, T., \& Pieters, C. M. 1992, in Proc. Lunar and Planetary Science, 22, 313

Hiroi, T., \& Pieters, C. M. 1994, Journal of Geophysical Research, 99, 10867 
Hiroi, T., Pieters, C. M., Zolensky, M. E., \& Lipschutz, M. E. 1994, in Proceedings NIPR Symposium No. 7, ed. K. Yanai, et al. (National Institute of Polar Research), 230

Hiroi, T., Zolensky, M. E., \& Pieters, C. M. 2001, Science, 293, 2234

Ishihara, D., et al. 2010, A\&A, 514, A1

Johnson, T. V., Yeates, C. M., \& Young, R. 1992, Space Science Reviews, 60, 3

Kasuga, T., et al. 2012, AJ, 143, 141

Kirkwood, D. 1867, Meteoric astronomy: a treatise on shooting-stars, fireballs, and aerolites., Philadelphia, J. B. Lippincott \& co.

Lagerkvist, C.-I., Moroz, L., Nathues, A., Erikson, A., Lahulla, F., Karlsson, O., \& Dahlgren, M. 2005, A\&A, 432, 349

Lazzarin, M., Marchi, S., Moroz, L. V., Brunetto, R., Magrin, S., Paolicchi, P., \& Strazzulla, G. 2006, ApJ, 647, L179

Lazzaro, D., Angeli, C. A., Carvano, J. M., Mothé-Diniz, T., Duffard, R., \& Florczak, M. 2004, Icarus, 172, 179

Lebofsky, L. A., et al. 1986, Icarus, 68, 239

Levison, H. F., Bottke, W. F., Gounelle, M., Morbidelli, A., Nesvorný, D., \& Tsiganis, K. 2009, Nature, 460, 364

Li, J.-Y., et al. 2010, Icarus, 208, 238

Mainzer, A., et al. 2011, ApJ, 731, 53

Masiero, J. R., et al. 2011, ApJ, 741, 68

Matson, D. L. 1971, in IAU Colloq. 12, Physical Studies of Minor Planets, ed. T. Gehrels (Washington: National Aeronautics and Space Administration SP-267), 45

Michałowski, T., et al. 2006, A\&A, 459, 663

Morbidelli, A., Jedicke, R., Bottke, W. F., Michel, P., \& Tedesco, E. F. 2002, Icarus, 158, 329

Mothé-Diniz, T. 2009, Icy Bodies of the Solar System, Proceedings IAU Symposium No. 263, ed. J.A. Fernaandez, et al., 231 
Mothé-Diniz, T., Carvano, J. M., \& Lazzaro, D. 2003, Icarus, 162, 10

Murakami, H., et al. 2007, PASJ, 59, S369

Neese, C., Ed., Asteroid Taxonomy V6.0. EAR-A-5-DDR-TAXONOMY-V6.0. NASA Planetary Data System, 2010.

Nesvorný, D., Jedicke, R., Whiteley, R. J., \& Ivezić, Z̆. 2005, Icarus, 173, 132

Neugebauer, G., et al. 1984, ApJ, 278, L1

Onaka, T., et al. 2007, PASJ, 59, S401

Ostro, S. J., et al. 2000, Science, 288, 836

Pravec, P., et al. 2012, Icarus, 221, 365

Rayman, M. D. 2003, Space Technology 23, 185

Russell, C. T., et al. 2004, Planetary and Space Science, 52, 465

Sasaki, S., Nakamura, K., Hamabe, Y., Kurahashi, E., \& Hiroi, T. 2001, Nature, 410, 555

Scholl, H., et al. 1989, in Asteroids II, ed. R. P. Binzel et al. (Tucson: University of Arizona Press), 845

Stern, A., \& Spencer, J. 2003, Earth, Moon, and Planets, 92, 477

Tedesco, E. F., Noah P. V., Noah, M., \& Price, S. D. 2002, AJ, 123, 1056

Tholen, D. J. 1984, PhD thesis, Arizona University

Tholen, D. J., \& Barucci, M. A. 1989, in Asteroids II, ed. R. P. Binzel et al. (Tucson: University of Arizona Press), 298

Thomas, C. A., Rivkin, A. S., Trilling, D. E., Marie-Therese E., \& Grier, J. A. 2011, Icarus, 212,158

Trieloff, M., et al. 2003, Nature, 422, 502

Usui, F., et al. 2011, PASJ, 63, 1117

Warner, B. D., et al. 2009, Icarus, 204, 172 
Weisberg, M. K., McCoy, T. J., \& Krot, A. N. 2006, in Meteorites and the Early Solar System II, ed. D.S. Lauretta \& H.Y. McSween (Tucson: University of Arizona Press), 19

Wright, E. L., et al. 2010, AJ, 140, 1868

Yoshikawa, M. 1989, A\&A, 213, 436

Zellner, B., \& Gradie, J. 1976, AJ, 81, 262

Zellner, B. 1979, in Asteroids (Tucson: University of Arizona Press), 783 